\title{
Soft Biomimetic Optical Tactile Sensing with the TacTip: A Review
}

\author{
Nathan F. Lepora
}

\begin{abstract}
Reproducing the capabilities of the human sense of touch in machines is an important step in enabling robot manipulation to have the ease of human dexterity. A combination of robotic technologies will be needed, including soft robotics, biomimetics and the high-resolution sensing offered by optical tactile sensors. This combination is considered here as a SoftBOT (Soft Biomimetic Optical Tactile) sensor. This article reviews the BRL TacTip as a prototypical example of such a sensor. Topics include the relation between artificial skin morphology and the transduction principles of human touch, the nature and benefits of tactile shear sensing, 3D printing for fabrication and integration into robot hands, the application of Al to tactile perception and control, and the recent step-change in capabilities due to deep learning. This review consolidates those advances from the past decade to indicate a path for robots to reach human-like dexterity.
\end{abstract}

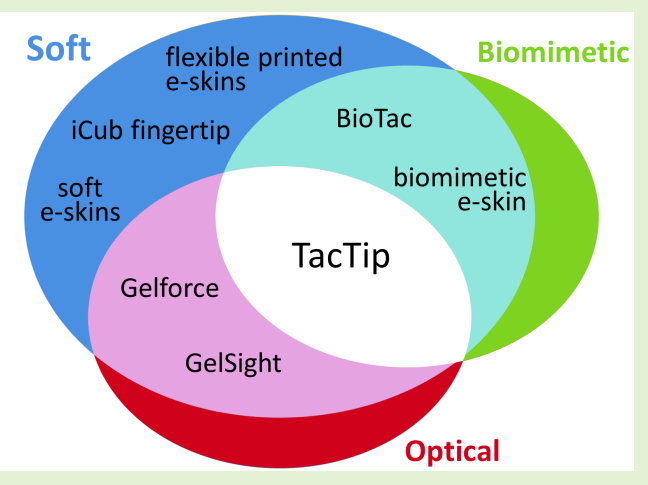

Index Terms-Force and tactile sensing, haptics, manipulation, robot dexterity, TacTip sensor

\section{INTRODUCTION}

As humans, we take our sense of touch for granted as we mostly use it in a subconscious way. Visual and auditory events grab our attention [1] while our tactile sense continues unabated during everyday manual tasks and chores. However, touch is arguably the sense that most differentiates humans from other animals because the dexterous use of our hands relies on the intelligent use of tactile perception. With our hands we have invented technology, the hallmark of our species, spanning from the archaeological record of archaic hominids to the modern era of advanced devices such as robots.

Reproducing the capabilities of the human tactile sense in machines is an important step in enabling robotic hands to reach the dexterity of the human hand. As argued compellingly by historian Yuval Noah Harrari in his book "Home Deus: A Brief History of Tomorrow", robot dexterity will have a profound impact on human society as machines become commonplace for physical labour [2]. This revolution in robot dexterity is beginning in the logistics industry, but promises broader impacts in manufacturing, health, construction, food production, recycling, conservation and renewable energy.

A range of robotic technologies will be needed to reach human-like dexterity in machines. Soft robotics is needed for safe devices that adapt according to the intelligence embodied in their materials and design. Biomimetics provides the only known example of a general-purpose manipulation device: the

This work was supported in part by Leverhulme Research Leadership Award on 'A biomimetic forebrain for robot touch' (RL-2016-39).

$\mathrm{N}$. Lepora is with the Department of Engineering Mathematics, Faculty of Engineering, University of Bristol and Bristol Robotics Laboratory, Bristol, UK. (e-mail: n.lepora@bristol.ac.uk). human hand and our manual intelligence to use it effectively. Intelligent interaction requires a rich source of contact information, as offered by high-resolution optical tactile sensors such as the MIT GelSight [3], [4] and BRL TacTip [5], [6] with deep learning methods such as convolutional neural networks.

Here, this combination of technologies is termed SoftBOT sensing, for Soft Biomimetic Optical Tactile Sensing, encompassing SoftBOT sensors, hands and robotic systems. The BRL TacTip uniquely combines all of these features as a prototypical example of a SoftBOT sensor, and for this reason has led to a large body of research on robot dexterity. Therefore, this article reviews tactile sensing with the TacTip to consolidate the advances with this technology over the past decade and so indicate a path to human-like robot dexterity.

\section{Soft, Biomimetic and Optical Tactile Sensing}

To motivate this article, we define the terms 'soft', 'biomimetic' and 'optical' in the context of tactile sensing, bearing in mind that a tactile sensor is a device that transduces deformation of a sensing surface into a signal containing information about the physical contact. As emphasised in past surveys [7]-[10], this information can be used to infer properties of the contacting object and its interaction with the sensor.

Soft tactile sensors rely on soft materials to elicit information from physical contact. While all tactile sensors use a deformable surface to sense, they range in material construction from flexible printed e-skins [11] to sensitive materials embedded within elastomeric substrates [12]. Softness can have many benefits in the design and function of tactile 
sensors. For example, it is important that e-skins are flexible so they can conform their sensing surface to $3 \mathrm{D}$ objects and cover large areas of hard or soft actuated robots [13].

Soft biomimetic tactile sensors are soft tactile sensors based on principles distilled from the study of biological systems [14], [15]. There is a distinction between 'blind copying', such as merely shaping a tactile sensor like a human fingertip, and true biomimicry, such as transferring the transduction principles of human skin into the design of an artificial sensor. Soft robots are often inspired by soft-bodied animals [16], so it is expected that biomimetic tactile sensors are usually soft. There are, however, many ways in which biological principles can motivate soft designs. One example is the inspiration for the Syntouch BioTac [17] from the multi-modality of human touch to pressure, vibration and temperature. Another example is a biomimetic e-skin that measures local shear and normal forces by reproducing the hill-like structure of the dermalepidermal boundary in human skin [18].

Soft optical tactile sensors are soft tactile sensors that use light to view the deformation. Optical tactile sensors are often soft because they rely on viewing physical changes to the sensing surface, usually from inside the sensor with an internal light source. Optical touch sensing dates back to the mid1960s [19], with the earliest example relaying the view of a photoelastic skin via optic fibres to a TV camera whose signal was viewed remotely to teleoperate a robotic gripper [20].

For soft camera-based tactile sensors, there are many proposals for imaging surface deformation [19] with categories including [21]: (i) soft marker-based optical tactile sensors, which typically measure the (lateral) shear deformation of the sensing surface, such as the GelForce (2004) which has markers embedded in a supporting gel [22]; and (ii) soft reflectionbased optical tactile sensors, which typically measure the (normal) indentation of the sensing surface, such as the GelSight (2009) which uses the surface shading from multiple internal light sources to infer a depth map via photometric stereo [3]. Combinations are also considered, such as by printing markers on the GelSight skin [23] and by mixing coloured markers to indicate depth [24]. Recently, this area of research has been boosted by the remarkable advances in image processing using convolutional neural networks [25], which has lead to many refinements of these designs [26]-[30].

Soft biomimetic optical tactile sensors combine optical imaging with biological principles underlying the sense of touch in animals. A technology that offers both optical and biomimetic tactile sensing is the BRL TacTip (centre of figure in graphical abstract), proposed in 2009 as 'a tactile sensor based on biologically-inspired edge encoding' [5]. This article will review the biomimetic and optical principles of the TacTip as a prototypical example of a SoftBOT sensor.

\section{Soft Biomimetic Optical Tactile Sensing: THE TACTIP}

The TacTip is a soft biomimetic optical tactile sensor that mimics the structure and function of the human fingertip (Figure 11. Human skin has an intricate morphology of layers, microstructures and sensory receptors that underlie its many functions, from protecting the body to sensing surface contact, temperature and injury [31]-[33]. Like most other mammals, our skin is of two general types: hairy over much of our body and glabrous (hairless) on the sensitive underside of our fingers and toes, palms, soles of our feet, external genitalia, areolae, tongue, inner cheeks and lips.

The shallow layers of glabrous skin are structured to sense touch via the deformation of its surface. These upper layers comprise an outer epidermis over an inner papillar dermis, which interdigitate in a mesh of dermal papillae and epidermal intermediate ridges (Figures $1 \mathrm{~A}, \mathrm{c}$ ). This 3D structure transmits shear and normal deformation of the skin surface into the displacement of sensory mechanoreceptors within the dermalepidermal interface. Thus, the dermal papillae and intermediate ridges are considered to act as a mechanical amplifier of skin deformation into mechanoreceptor activity [44].

The TacTip design is based on the shallow layers of glabrous skin [5], [6]. It has an outer biomimetic epidermis made from a rubber-like material over a soft inner biomimetic dermis made from an elastomer gel (Figures $1 \mathrm{p}, \mathrm{d}$ ). These two materials interdigitate in a mesh of biomimetic intermediate ridges and dermal papillae, comprising stiff inner nodular pins that extend under the biomimetic epidermis into the soft gel. This structure amplifies surface deformation of the skin into lateral movement of visible markers on the pin tips.

In human skin, two types of sensory mechanoreceptor are embedded within the dermal-epidermal interface: Merkel cells on the intermediate ridges and Meissner corpuscles within the dermal papillae (Figure 1 $1 \mathrm{c}$ ). Merkel cells respond to skin deformation, activating slowly-adapting (SA) neurons that fire during sustained contact, such as when perceiving shapes or edges. Meissner corpuscles respond to skin movement, activating rapidly-adapting (RA) neurons that fire during changes of contact, such as when perceiving surface slip and flutter. These sensory receptors work together to convey information about the tangible aspects of touch [45].

The biomimetic counterparts to these sensory mechanoreceptors are the markers on the pin tips, which can be imaged through a transparent gel forming the dermis (Table I . The marker displacements from rest are analogous to the SA activity from the Merkel cells because they indicate sustained deformation of the sensing surface [40] to enable accurate shape and edge recognition [34], [46]. Likewise, the counterpart of RA mechanoreceptor activity from the Meissner corpuscles are the marker velocities, because these comprise a transient signal that indicates skin motion. Accordingly, the marker velocities enable accurate slip detection [35], [36]. Together, these two signals - the marker displacements and velocities - give information about the sustained deformation and transient motion of the sensing surface.

The deeper layers of glabrous skin also have biomimetic counterparts in the TacTip design (Table I). Skin maintains its compliance from the deep reticular dermis and subcutaneous fat (Figure 1 $1 \mathrm{a}$ ), whose biomimetic counterpart is the deeper elastomer gel held in place by a transparent acrylic cap. Within the deep skin layers, a population of RA mechanoreceptors called Pacinian corpuscles responds to high-frequency vibration (peak sensitivity $\sim 250 \mathrm{~Hz}$ ). A partial mimicry of their 
(a) Skin physiology

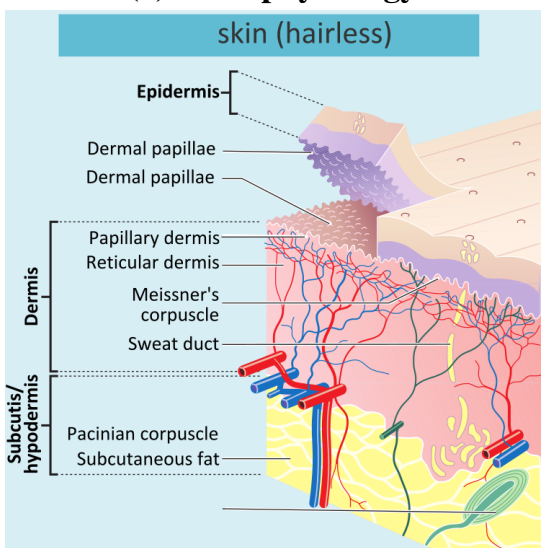

(c) Skin transduction

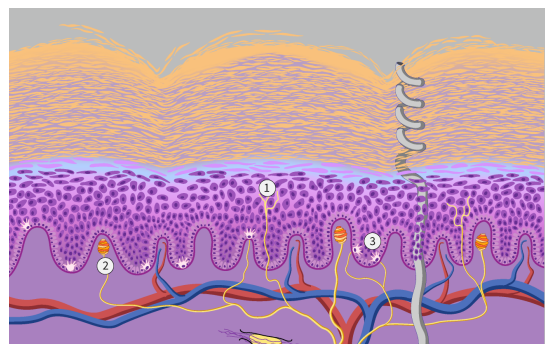

(b) Biomimetic tactile sensor

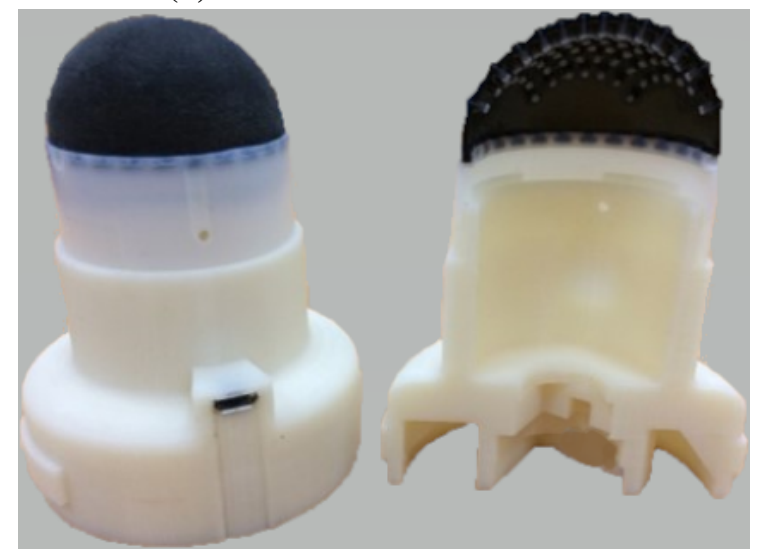

(d) Biomimetic transduction

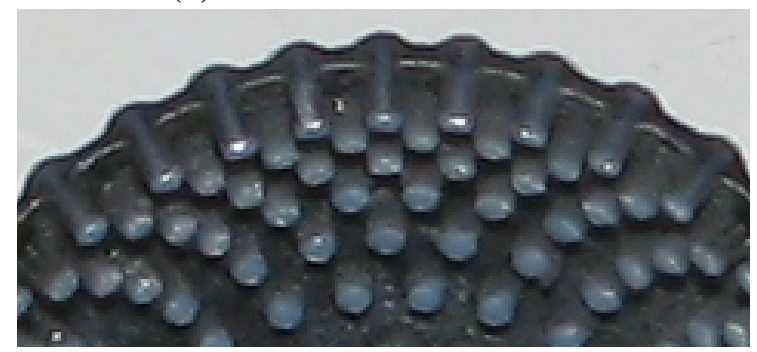

Fig. 1. Biomimetics of the TacTip. (a) Diagram of the layered morphology of hairless skin; (b) Cut-through of the 3D-printed BRL TAcTip (2016/18); (c) Close-up of the interdigitation of dermis and epidermis, with sites of mechanoreceptors; (d) Close-up of a cut-through of the TacTip skin. The morphology of the artifical skin is based on natural skin. (Credits: Wikipedia, 'Skin Layers', 'Hegasy skin layers Receptors', CC By-SA License.)

\begin{tabular}{c} 
Neurophysiology \\
\hline epidermal ridges \& dermal papillae \\
reticular dermis \& subcutaneous fat \\
SA-I mechanoreceptors (Merkel cells) \\
RA-I mechanoreceptors (Meissner corpuscles) \\
RA-II mechanoreceptors (Pacinian corpuscles) \\
nociceptors (free nerve endings) \\
thermoceptors (free nerve endings) \\
overlapping sensitive receptive fields \\
epidermal ridges (fingerprint) \\
neural spiking
\end{tabular}

Function
transmits \& amplifies deformation of surface to mechanoreceptors
soft structure \& compliance
sense sustained skin deformation; perception of shape \& edges
sense transient skin movement; perception of flutter \& surface slip
vibration sensing; perception of surface texture
noxious touch
temperature difference sensing
hyperacuity
friction \& improved transduction; induces incipient slip
efficient signal encoding

TABLE I
Biomimetic counterpart pins \& markers [5], [6] elastomer gel [5], [6] pin displacements [6], [34] pin velocities [35], [36] under investigation [37], [38] under investigation [38] thermoactive skin [39] super-resolution [40]

3D-printed fingerprint [36], [37], [41] event-based imaging [42], [43]

BIOMIMETICS OF THE TACTIP, MATCHING THE NEUROPHYSIOLOGY AND FUNCTION.

function can be attained by using the TacTip with a high frame-rate $(\mathrm{kHz})$ camera [37], [38]; however, questions remain about whether this approach to vibration sensing is effective or even biomimetic, since it images fast pin movement rather than vibration in the deeper gel. In our view, a biomimetic counterpart of the vibration sense would be to embed a pressure sensor in the gel of the TacTip, like the vibration modality of the BioTac [17]. Other tactile sensing modalities can also be included, such as temperature sensing by using a thermoactive smart material for the outer TacTip skin, which is imaged as a background to the markers [39].

A consequence of the biomimetic design of the TacTip is that other properties of human perception emerge. An important aspect of human tactile perception is hyperacuity: a capacity to discriminate extended spatial features to a submillimetre acuity that is finer than the millimetre-scale spacing between mechanoreceptors [47]. The TacTip also exhibits tactile hyperacuity, with a sub-millimetre capacity for spa- tial discrimination that is finer than its millimetre-scale pin spacing [40]. Fundamentally, the hyperacuity arises because both the biological and artificial tactile senses are comprised of arrays of overlapping, broad but sensitive receptive fields. This structure enables spatial interpolation over neighbouring receptors, which is analogous to a well-known technique in optical imaging known as super-resolution [48].

Perhaps surprisingly, the role of the human fingerprint in the sense of touch is still being investigated [49] after two centuries of study. A 3D-printed fingerprint can be reproduced in the TacTip as raised bumps [37], [41] or concentric raised rings over the papillae [50]. Benefits of a biomimetic fingerprint include increased sensitivity to texture [37] and spatial localisation [41]. A ringed biomimetic fingerprint can also induce incipient slip [50], where a local region of skin slips in detectable way before the grip slips [35], [36].

Finally, another direction to take biomimetic optical tactile sensing is to adopt neuromorphic transduction using trains 
of 'spike' events [51], [52]. Biological nervous systems use event-based signalling to represent sensory information in an efficient and temporally-precise way, which neuromorphic engineering aims to reproduce artificially [53]. A benefit of optical tactile sensing with the TacTip is that an event-based camera can be used to implement a transduction mechanism that has both a biomimetic skin design and is neuromorphic from the camera [42], [43]. Overall, neuromorphic computation offers a new paradigm for robot touch that complements the capabilities of soft biomimetic optical tactile sensors.

\section{TActile Shear SEnsing}

\section{A. Tactile sensing of normal strain and shear strain}

A central aspect of tactile sensing with the TacTip is that it measures contact via shear. Skin indentation tilts and moves the nodular pins, causing lateral displacements of markers on the pin tips (Figure 2 $\mathrm{b}$, right). The biomimetic structure of stiff nodular pins interdigitating with a soft elastomeric gel causes mechanical transduction of normal-strain on the skin surface into a measurable shear-strain underneath the skin.

This design also makes the TacTip highly sensitive to shearstrain of the skin surface. The two sources of skin movement, normal- and shear-strain, produce distinct patterns of marker displacement. Sliding or slip gives a more uniform shearstrain field, whereas normal indentation can produce characteristic dipole or multipole patterns (Figure $2 \mathrm{a}$ ). In practise, both normal- and shear-strain are usually present, which can introduce subtleties when inferring contact shape information from the TacTip, as discussed later.

Therefore, the TacTip operates in a distinct manner from the common types of taxel-based artificial tactile sensors that measure normal-strain, such as the BioTac [17] and the iCub fingertip [54]. The TacTip is also distinct from other designs of optical tactile sensor. Reflection-based optical tactile sensors such as the GelSight [3] reconstruct normal-strain directly from light shading. Some optical tactile sensors also use markers coated underneath the skin [55] (including recent GelSight versions [23]), but those measure just the shear-strain of the skin surface. In contrast, the TacTip markers inform about both the normal- and shear-strain due to contact.

Several optical tactile sensors use markers floating in the elastomer underneath the outer skin, such as the GelForce [22], [56] and ChromaTouch [24]. These tactile sensors differ from the TacTip by not having stiff nodular pins connecting the deformation of the skin surface to the markers, but instead rely on the forces transmitted through the soft elastomer. The GelForce uses two layers of coloured spherical markers in a transparent elastomer to infer force vectors within the gel [22], [56], and the ChromaTouch uses two layers of partiallytransparent coloured markers whose movement and colourmatching informs about the sensor deformation [24].

All these designs have their pros and cons as artificial tactile sensors. The overall point to emphasise is that the TacTip senses contact differently from other tactile sensors and uses a biomimetic mechanism for this transduction.

\section{$B$. The shear-sensing hypothesis}

Platkiewicz, Lipson and Hayward have emphasised the importance of internal shear strain for sensing external contact [57] with their shear-sensing hypothesis: 'we propose that shape-related tactile information is more suitably recovered from shear strain than normal strain'.

Their reasoning is based on the contact mechanics of touch: 'the pressure distribution at the surface of a tactile sensor cannot be acquired directly and must be inferred from the [strain] field induced by the touched object in the sensor medium.' In vision, it is well known that edge detection is fundamental to processing shape, with the convolutions of deep neural networks analogous to edge-detecting neurons in visual cortex. For touch, there are two ways to detect edges: indirectly from gradients of the normal-strain field or directly from zero crossings (sign changes) of the internal shear-strain field (Figure $2 \mathrm{p}$ ). Zero crossings are a more robust measure of shape from touch, because they are affected less by distortion from the mechanics of skin and require no computation of signal gradients [57], motivating the use of shear strain.

This reasoning can be grounded in a biomechanical model of skin [57] that approximates the shear-strain field $\gamma$ at depth $z$ and horizontal position $x$ in a uniform elastomer (Young's modulus $E$ ) due to a pressure field $p(x)$ at the surface:

$$
\gamma(x, z) \approx \frac{z}{E / 3} \frac{\mathrm{d}}{\mathrm{d} x} p_{\epsilon}(x, z), \quad p_{\epsilon}(x, z)=p(x) * \phi_{\epsilon}(x, z),
$$

where $p_{\epsilon}(x, z)$ represents that the pressure field is mechanically blurred by depth (modelled by convolving a Gaussian $\phi_{\epsilon}(x)$ of width $\epsilon(z)$ that increases with depth). Overall, the shear-strain field follows the gradient of the (blurred) pressure profile and increases linearly with depth from zero at the surface - i.e. it has mechanically calculated the signal gradient.

However, the skin of the TacTip is not a uniform elastic material, but has an inner structure of stiff nodular pins interdigitating with soft elastomeric gel. Hence, this model (1) is more suited to optical tactile sensors with floating markers such as the GelForce and ChromaTouch [22], [56]

A biomechanical model of the TacTip and the dermal papillae should be based instead on the levering of the nodular pins caused by the normal skin indentation $\delta(x)$ due to surface pressure. Simply using the geometry of a pin angled normal to the gradient of the skin indentation field (Figure $2 \mathrm{~b}$ ), gives:

$$
\gamma(x, z)=z \sin \arctan \left(\frac{\mathrm{d}}{\mathrm{d} x} \delta(x)\right) \approx z \frac{\mathrm{d}}{\mathrm{d} x} \delta(x),
$$

for pins of length $z$ forming the hypotenuse of a right-angled triangle with shear-strain along the (horizontal) adjacent side. For shallow gradients, $\frac{\mathrm{d}}{\mathrm{d} x} \delta(x) \ll 1$, the small angle approximation gives a shear-strain field that is linear in the gradient of the indentation profile; i.e. also calculates the signal gradient.

It is interesting that these two biomechanical models lead to very similar equations: in both, the shear-strain field $\gamma(x, z)$ is proportional to depth $z$ and the gradient of a quantity (pressure or indentation) at the skin surface. Both models amplify the transduction with depth $z$, but the pin structure is beneficial in not being mechanically blurred $\epsilon(z)$ compared to 
(a) Single tactile image processed into shear-strain field (coloured by strain)

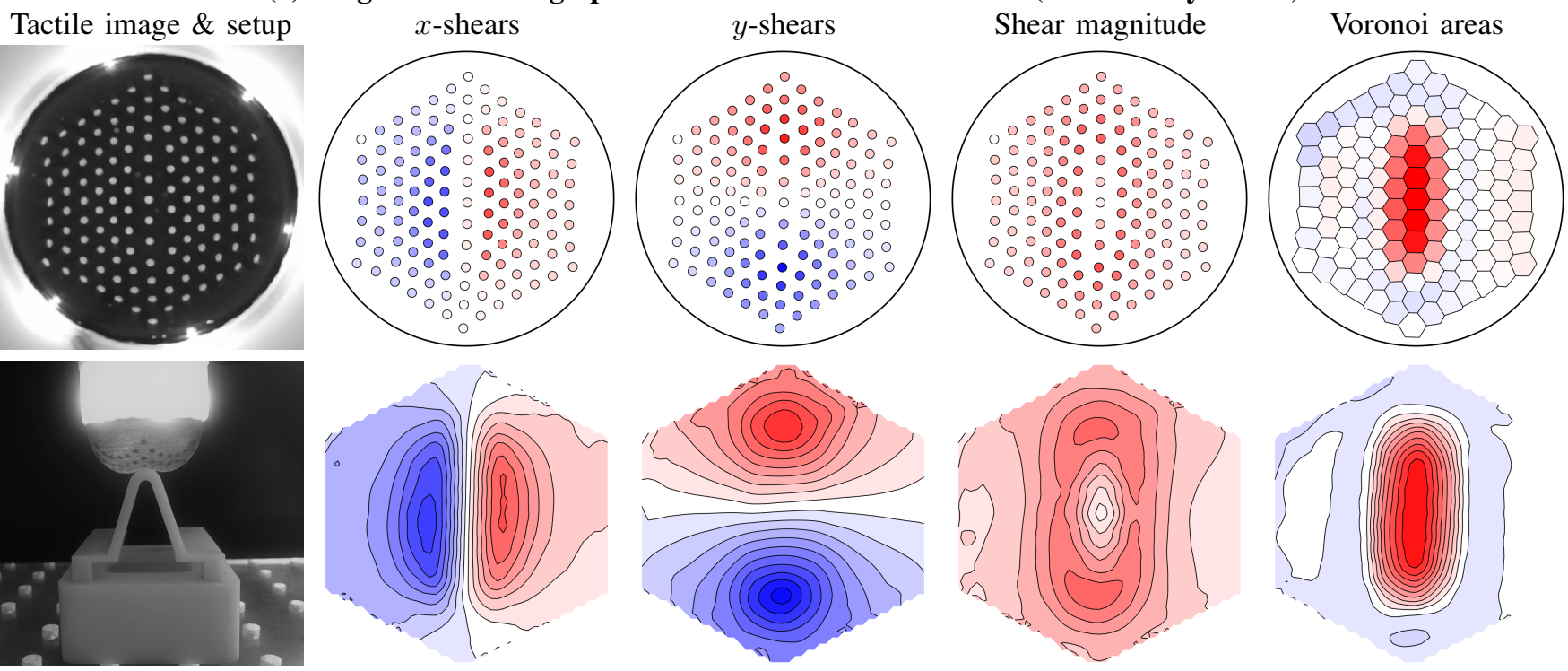

(b) Biomimetic signal transduction

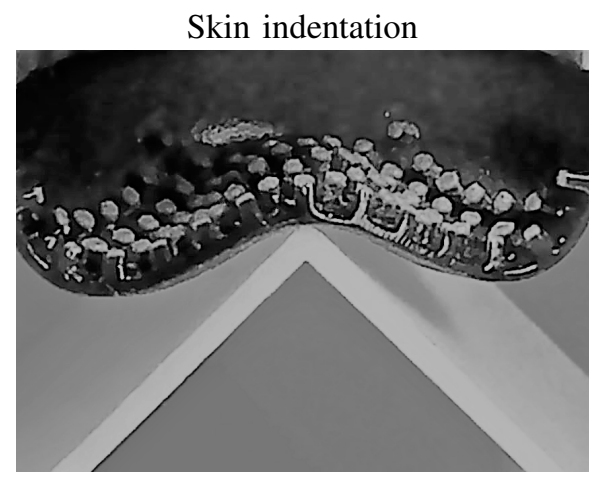
Levering of pins
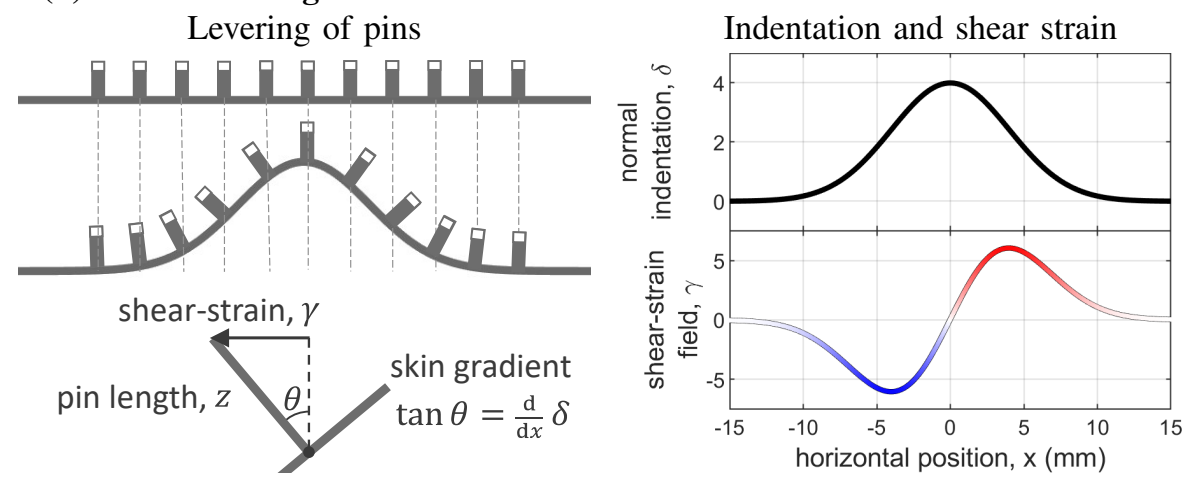

(c) Time series of tactile images processed into shear strains (coloured by marker)
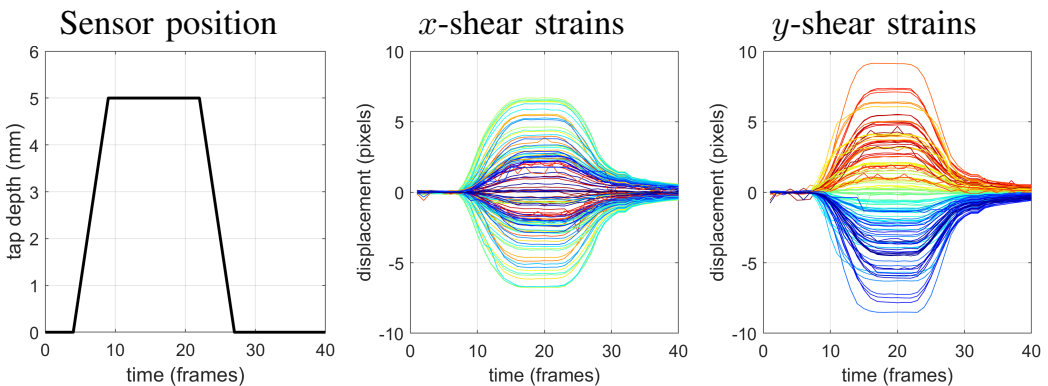

Shear-strains
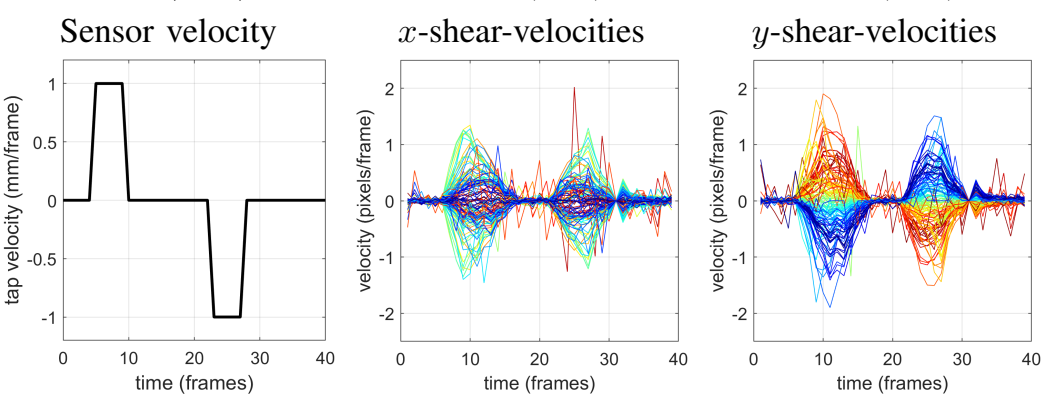

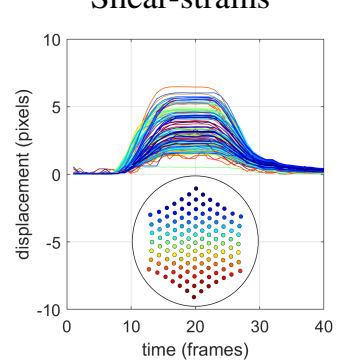

Shear-velocities

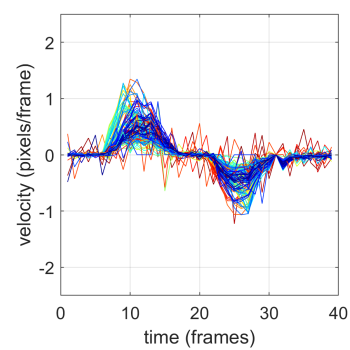

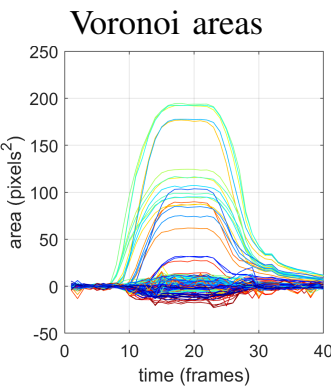

Voronoi area changes

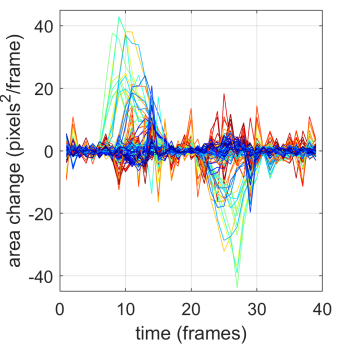

Fig. 2. Sensing shape with the BRL TacTip. (a) Processing of a tactile image of an edge contact (left) into 2D ( $x, y)$-shear strains, from whih shear magnitude and Voronoi area change are extracted (red/blue indicates positive/negative change). (b) Biomimetic signal transduction of skin indentation into marker displacement as a shear-strain field. (c) Time series of quantities in panel (a) and their derivatives for a discrete tap onto then off an edged stimulus (marker labelled by colour). This biomimetic representation using shear-strain is highly informative about the contact. 
the elastomer. Also, the shear-strain from the stiff pins should be larger than that of an elastic medium.

Overall, the reasoning behind the shear-sensing hypothesis appears consistent with both the uniform elastomeric model considered originally (Equation 1) and the pin model introduced here for the TacTip (Equation 22. The TacTip is unique amongst related tactile sensors by sensing touch purely from shear via a mechanism of stiff pins to transduce skin indentation into shear strain. Therefore, the shear-sensing hypothesis appears to encapsulate the operation of the TacTip as an embodiment of the theory.

\section{Period I (2009-2014): INITIAL DeVelopment of THE TACTIP}

Most of the research and development into soft biomimetic optical tactile sensing has been within the Bristol Robotics Laboratory (BRL) in the U.K. However, the focus has moved from soft to medical to tactile robotics, leading to renewal of the underlying technology and research direction. Meanwhile, the field of tactile robotics has risen in prominence since the TacTip was introduced in 2009 as the aim of the field to enable artificial manipulation has become closer to reality. Recently, the revolution in deep learning [25] has raised the prominence of optical tactile sensors because of their compatibility with convolutional neural networks, promising an entirely new level of robot performance approaching human dexterity.

The first period of development (2009-2014) encompassed the original design for an optical tactile sensor based on biologically-inspired encoding [5], [58]. The name TACTIP was coined [37], [59] as a contraction of 'tactile fingertip' after an EU-funded project that supported some of the research. The name has stuck, but is now written TacTip similarly to other well-known tactile sensors (e.g. BioTac or GelSight).

The inspiration for the TacTip was the tactile contact lens [60], [61], developed in a Toyota-funded research laboratory to help humans feel manufacturing defects in automobile production. The tactile contact lens magnifies the feeling of surface deformation using a slippery flexible base-plate attached to an array of rigid pins held against a human fingertip (Figure 3). As the base slides over a small bump or crack, the pins then act as levers to amplify the normal indentation in the base into shear-strain that a human can feel [62]. Overall, the tactile contact lens magnifies imperceptible surface contacts into larger shear displacements by about a factor of four, which can be discriminated more easily.

The TacTip uses this same mechanical principle to magnify indentations on a flexible outer skin into lateral movement of markers on the pin tips [5]. Both the TacTip and tactile contact lens are biomimetic, because this mechanism for magnifying surface contact into shear-strain seems to have evolved in human tactile skin: the human sense of touch relies on the mechanical structure of dermal papillae and epidermal intermediate ridges, as explained in Section III.

A central question when using any soft optical tactile sensor is how to interpret the tactile image from the camera to infer aspects of the skin deformation (Figure 2). Initial studies with the TacTip processed the images into velocity

\section{(a) Mechanics of the Tactile Contact Lens}
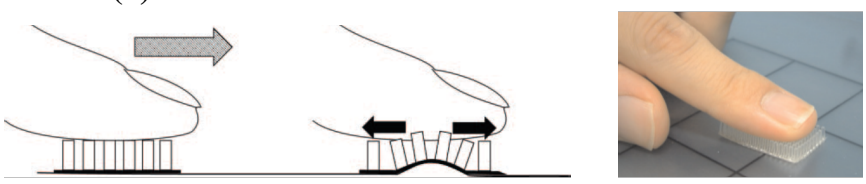

(b) Mechanics of the Human Fingertip and TacTip
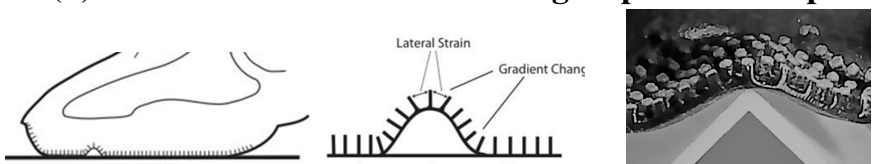

Fig. 3. Analogous operation of the Tactile Contact Lens and TacTip. Both devices magnify and transduce surface indentation into shear strain, using the levering of an array of pins.

vector fields of marker motion, taking inspiration from the Meissner corpuscles embedded in the dermal papillae which are motion-sensitive [5], [58]. Surface edge features such as the contours of a coin were clearly visible in the marker velocity field, motivating the initial research on biologicallyinspired edge encoding. Other early work used image filtering (dilation/erosion) to transform a tactile image into a visualisation of marker density showing the orientation of a contacted edge [63], [64]. Other simple methods to infer contact features were also explored, such as binning the tactile image into a discrete array, which was applied to lump detection and localization in medical haptics [65]-[67].

Refinements of the original TacTip design were considered for an EU-funded project that sought to address the need for tactile robot manipulators and probes for industrial and healthcare applications. The tactile tip was miniaturized to a $20 \mathrm{~mm}$ diameter dome (from $40 \mathrm{~mm}$ ) for integration as a fingertip of an anthropomorphic robot hand [59] and a high frame rate camera used to investigate texture perception [37]; however, in both cases the camera was separate rather than integrated into the design. These early studies laid the foundation for later advances that used 3D printing to improve the TacTip.

\section{PERIOD II (2015-): THE TACTIP FAMILY}

The second period of the TacTip development (2015present) introduced some common approaches that allowed research on soft biomimetic optical tactile sensing at BRL to build into a coherent body of work. Multi-material 3D printing was adopted for fabrication alongside modular design principles [6], which encouraged diversification into a family of tactile sensors, hands and robotic systems (Figure 47. A common method based on tracking the markers was used for tactile image processing [34], [40], so the data could be treated as multi-dimensional time series for application of standard machine learning methods. These developments enabled the TacTip to be integrated into complete robotic systems that perceive, explore and manipulate their environments.

\section{A. 3D-printed TacTip and integration into robot hands}

The adoption of multi-material 3D printing for TacTip manufacture [6] was key to developing a family of rapidlyprototyped tactile probes, grippers and manipulators (Fig- 


\begin{tabular}{|c|c|c|}
\hline Sensor & Year & Design \\
\hline TACTIP [5] & 2009 & $\begin{array}{l}\text { cylindrical body ( } 40 \mathrm{~mm} \text { dia. spacer) } \\
\text { molded hemispherical soft tip ( } 40 \mathrm{~mm} \text { dia., } 532 \text { pins) }\end{array}$ \\
\hline Mini TACTIP [59] & 2012 & $\begin{array}{l}\text { rigid 3DP mount on digit of Elumotion robotic hand } \\
\text { molded hemispherical soft tip ( } 20 \mathrm{~mm} \text { dia., } 276 \text { pins) }\end{array}$ \\
\hline Open TacTip [68] & 2016 & $\begin{array}{l}\text { rigid 3DP body }(161 \mathrm{~mm}) \text { modified for modular tip } \\
\text { molded hemispherical soft tip ( } 40 \mathrm{~mm} \text { dia., } 532 \text { pins) }\end{array}$ \\
\hline TacTip [6] & 2016 & $\begin{array}{l}\text { rigid 3DP body }(85 \mathrm{~mm}) \text { and modular tip } \\
\text { hemispherical 3DP soft tip ( } 40 \mathrm{~mm} \text { dia., } 127 \text { pins) }\end{array}$ \\
\hline TacTip-M2 [69] & 2016 & $\begin{array}{l}\text { integrated as digit of 3DP M2 gripper } \\
\text { rectangular 3DP soft finger }(32 \times 102 \times 44 \mathrm{~mm} ; 80 \text { pins })\end{array}$ \\
\hline TacCylinder [70] & 2017 & $\begin{array}{l}\text { 3DP body and soft skin } \\
\text { cylinder ( } 63 \mathrm{~mm} \text { dia., } 82 \mathrm{~mm} \text { length, } 180 \text { pins })\end{array}$ \\
\hline TacTip-GR2 [71] & 2017 & $\begin{array}{l}\text { integrated as } 2 \text { fingertips of 3DP GR2 gripper } \\
\text { 3DP fingertip ( } 40 \mathrm{~mm} \text { dia. } \times 44 \mathrm{~mm} \text { depth, } 127 \text { pins })\end{array}$ \\
\hline TacTip-FP1 [41] & 2017 & $\begin{array}{l}\text { modified tip with 'fingerprint' (raised bumps) } \\
\text { hemispherical 3DP soft tip ( } 40 \mathrm{~mm} \text { dia., } 127 \text { pins) }\end{array}$ \\
\hline TacTip-SYM [72] & 2017 & $\begin{array}{l}\text { modified tip with } 12 \text {-fold rotational symmetry } \\
\text { hemispherical 3DP soft tip ( } 40 \mathrm{~mm} \text { dia., } 49 \text { pins })\end{array}$ \\
\hline TacTip-DM [38] & 2018 & $\begin{array}{l}\text { customized TacTip for optical flow sensor } \\
\text { small hemispherical 3DP soft tip ( } 28 \mathrm{~mm} \text {; } 19-61 \text { pins) }\end{array}$ \\
\hline MultiTip [39] & 2018 & $\begin{array}{l}\text { modified tip moulded from thermoactive material } \\
\text { body similar to } 2009 \text { TACTIP ( } 40 \mathrm{~mm} \text { dia., } 127 \text { pins) }\end{array}$ \\
\hline TacWhisker [73] & 2018 & $\begin{array}{l}\text { modified tip with } 193 \mathrm{DP} \text { whiskers fitting into pins } \\
\text { also a tendon-actuated version with } 2 \times 5 \text { whiskers }\end{array}$ \\
\hline TacTip [35] & 2018 & same as 2016 version \\
\hline TacTip-SMG [74] & 2019 & $\begin{array}{l}\text { integrated as } 3 \text { fingertips of Shadow Modular Grasper } \\
\text { custom 3DP soft fingertips }(\sim 40 \times 40 \times 44 \mathrm{~mm}, 100 \text { pins })\end{array}$ \\
\hline TacTip-Mini [75] & 2020 & $\begin{array}{l}\text { modified tip with small area and high pin density } \\
\text { small domed 3DP soft tip ( } 25 \mathrm{~mm} \text { dia., } 331 \text { pins })\end{array}$ \\
\hline TacTip-FP2 [36] & 2020 & $\begin{array}{l}\text { modified tip with fingerprint to induce incipient slip } \\
\text { ridged 3DP soft tip ( } 40 \mathrm{~mm} \text { dia., } 44 \text { pins) }\end{array}$ \\
\hline TacFoot [76] & 2020 & $\begin{array}{l}\text { integrated as foot of Lynxmotion SQ3U quadruped } \\
\text { small hemispherical 3DP soft tip ( } 28 \mathrm{~mm} ; 37 \text { pins) }\end{array}$ \\
\hline NeuroTac [42] & 2020 & $\begin{array}{l}\text { neuromorphic TacTip with event-based output } \\
\text { hemispherical 3DP soft tip ( } 40 \mathrm{~mm} \text { dia, } 61 \text { pins) }\end{array}$ \\
\hline NeuroTac-SoftH & & $\begin{array}{l}\text { integrated as fingertip of Pisa/IIT SoftHand } \\
\text { small 3DP soft fingertip }(20 \times 25 \times 30 \mathrm{~mm} ; 38 \text { pins })\end{array}$ \\
\hline TacTip-O [50] & 20 & $\begin{array}{l}\text { integrated as } 3 \text { fingertips of 3DP Model-O hand } \\
\text { small 3DP soft fingertips }(20 \times 40 \times 35 \mathrm{~mm}, 30 \text { pins })\end{array}$ \\
\hline TacTip-SoftH [30] & 2021 & $\begin{array}{l}\text { integrated as fingertip of Pisa/IIT SoftHand } \\
\text { small 3DP soft fingertip }(12 \times 19 \times 17 \mathrm{~mm} ; 35 \text { pins })\end{array}$ \\
\hline TacTip & 2021 & $\begin{array}{l}\text { rigid 3DP body ( } 45 \mathrm{~mm}) \text { and modular tip } \\
\text { hemispherical 3DP soft tip ( } 40 \mathrm{~mm} \text { dia., } 330 \text { pins) }\end{array}$ \\
\hline
\end{tabular}

LifeCam VX webcam, 480p 30fps, $f \approx 50 \mathrm{~mm}$

Not integrated

LifeCam Cinema webcam, 720p 30fps, $f \approx 50 \mathrm{~mm}$

LifeCam Cinema board (disassembled camera)

LifeCam Cinema board

Catadioptric $360^{\circ}$ lens; LifeCam Cinema HD

Raspberry Pi Spycam, Fisheye lens, $f \approx 20 \mathrm{~mm}$

LifeCam Cinema board

LifeCam Cinema board

ADNS-3080 dual mode, $30 \times 30$ pix $3 \mathrm{fps} / 6.4 \mathrm{kHz}$

LifeCam VX webcam, 480p 30fps, $f \approx 50 \mathrm{~mm}$

LifeCam Cinema board

ELP camera module, 1080p 30fps - 360p 120fps

ELP camera module; wide angle lens $f \approx 20 \mathrm{~mm}$

ELP camera module

ELP camera module

Hydream USB endoscope, 480p 30fps $\mathrm{f} \approx 40 \mathrm{~mm}$

Inivation DVS240, 240×120pix 12Mevents/s

Inivation mini-eDVS, $128 \times 128$ pix 0.6 Mevents/s JeVois Vision module

$1280 \times 1024$ pix 15 fps to $276 \times 144$ pix $120 \mathrm{fps}$

Misumi Model SYD, 1080p 60fps $\mathrm{f} \approx 10 \mathrm{~mm}$

ELP camera module; wide angle lens $f \approx 10 \mathrm{~mm}$

\section{TABLE II}

TACTIP FAMILY OF SOFT BIOMIMETIC OPTICAL TACTILE SENSORS. KEY: 3D-PRINTED (3DP), $640 \times 480$ PIX (480P), 1280×720 PIX (720P), $1920 \times 1080$ PIX (1080P), FRAMES PER SECOND (FPS), FOCAL LENGTH $(\boldsymbol{f})$. LIFECAM CCD WEBCAMS BY MICROSOFT, ELP (AILIPU

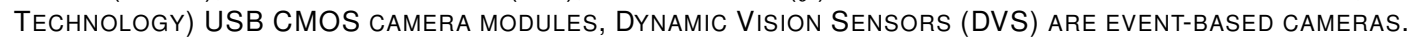

\begin{tabular}{cccc} 
Hand & DoA & Fingers & Examined capabilities \\
\hline Tactile Model M2 (T-M2) & 1 & 2 (1 tactile) & precise rolling manipulation [6], [69] \\
Tactile Model GR2 (T-GR2) & 2 & 2 tactile & recise rolling manipulation [6], [71] \\
Tactile Model O (T-MO) & 4 & 3 tactile & retains grasping capabilities; tactile object recognition; grasp success prediction [50] \\
Tactile Modular Grasper (T-MG) & 9 & 3 tactile & slip detection \& correction; light grasping on first attempt [36] \\
manipulation to desired grasp [74] & sensorimotor control of touch [30]
\end{tabular}

TABLE III

INTEGRATION OF THE TACTIP INTO ROBOT HANDS VARYING IN DEGREES OF ACTUATION (DOA) AND NUMBERS OF FINGERTIPS.

ure 47. The soft biomimetic optical tactile sensor was redesigned (2016/2018 TacTip; Table IIT) to have a compact modular base housing the circuit board from a web-camera; likewise, the tip was 3D-printed in one piece combining a flexible skin joined to papillae tipped with rigid white markers [6]. This redesigned TacTip became the standard device used in BRL for tactile sensing research. The 3Dprinted skin is robust to laboratory testing, with the tip only needing replacing after human error in operating industrial robot arms (even then, small tears have been repaired).

3D-printing also enabled the sensor to be customised for diverse applications, from creating a tactile sensing foot for walking robots [76] to mimicking rodent tactile whiskers [73]
(Figure 4, bottom row). A more biomimetic version of the TacTip skin with raised bumps over the pins (like a fingerprint) and increased dermal-epidermal stiffness contrast (rigid cores to the pins) improved the spatial acuity of the tactile sensor [41]. Further progression to a ringed biomimetic fingerprint helped induce and detect incipient slip, by encouraging the outer contact region to move before global slip occurs, giving sufficient time to react before losing the grasp [78].

These advances in soft tactile sensors complement the rapid progress in 3D-printed robot hands, examplified by the Yale OpenHand Project: a library of low-cost 3D-printed underactuated hand designs [79]. These tendon-driven, 2-4 fingered compliant hands have an underactuated adaptability 


\section{SoftBOT Sensors: Soft Biomimetic Optical Tactile Sensors}
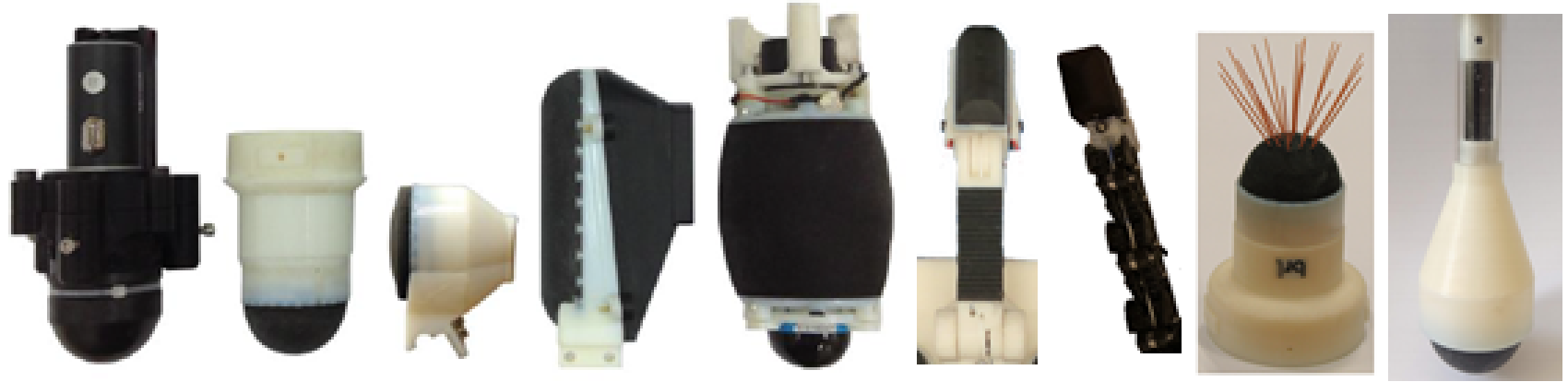

SoftBOT Hands: Soft Biomimetic Optical Tactile Hands
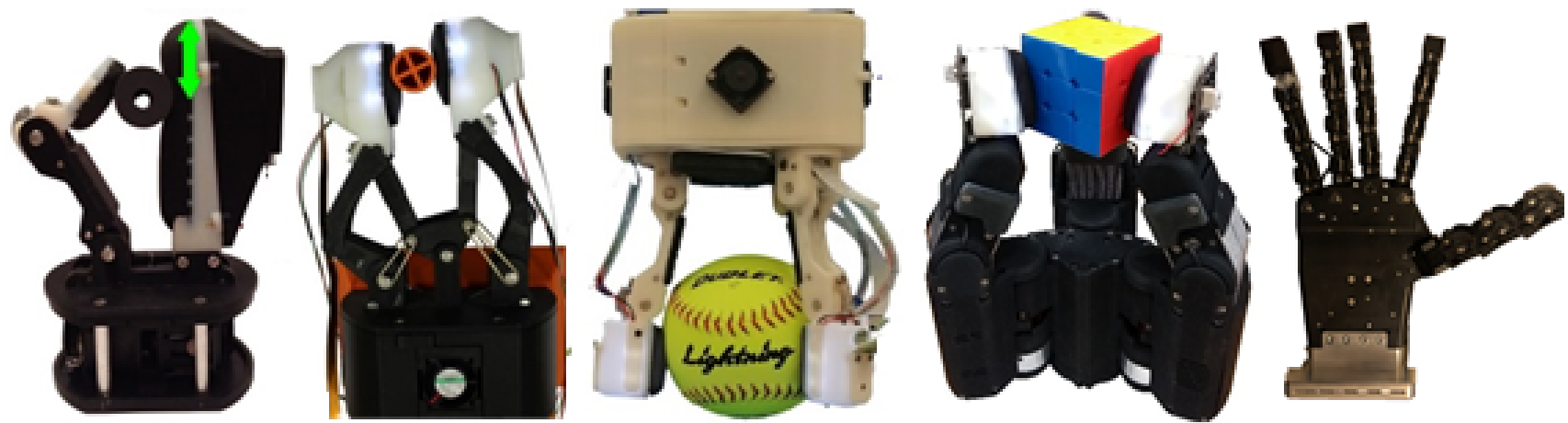

SoftBOT Systems: Soft Biomimetic Optical Tactile Systems
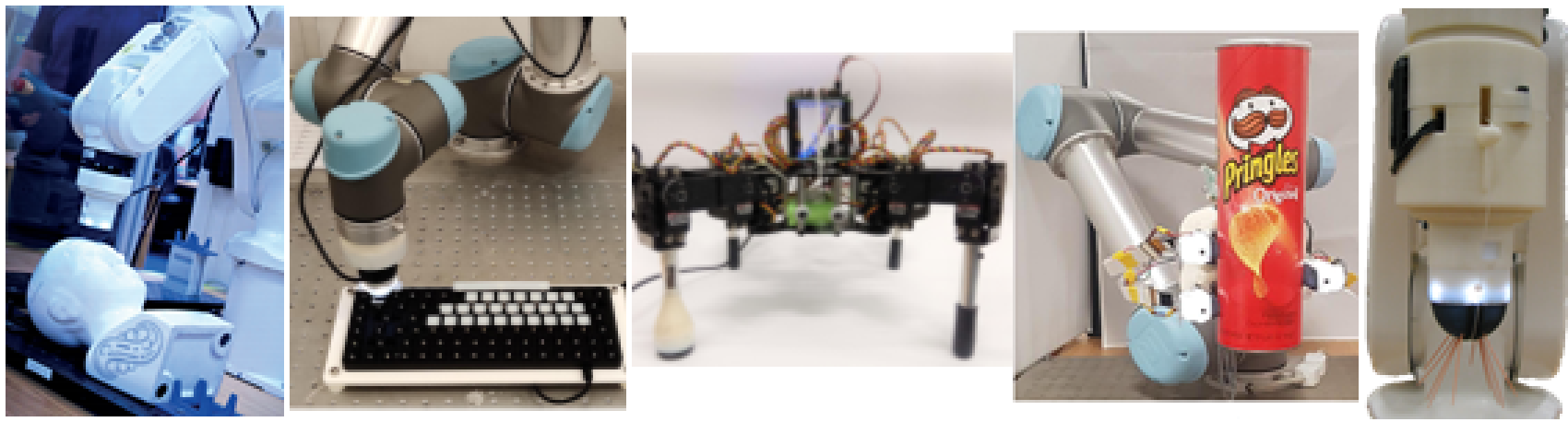

Fig. 4. SoftBOTS from the TacTip family. SoftBOT sensors (top row): original 2009 TACTIP [5], 3D-printed TacTip [6], TacTip-GR2 [71], TacTipM2 [69], TacCylinder [70], TacTip-O [50], TacTip-SoftHand [30], TacFoot [76] and TacWhisker [73]. SoftBOT hands (middle row): tactile ModelM2 [69], tactile Model-GR2 [71], tactile Model-O [50], tactile Shadow Modular Grasper [74] and tactile SoftHand [30]. SoftBOT systems (bottom row): 3D-printed TacTip on ABB robot arm for tactile servo control [46], [77], TacTip on UR5 arm for reinforcement learning [75], TacFoot on walking robot [76], tactile Model-O on UR5 robot arm for grasping and slip detection [36], [50] and tacWhisker mounted on ABB robot arm [73].

that passively conforms their grasping to a wide range of object geometries using only open-loop control. The most well-known is the 3-fingered Model O, based on the iHY (iRobot-Harvard-Yale) Hand [80] from the DARPA Robotic Manipulation-Hardware (ARM-H) program, which has been commercialised as the Reflex Hand (RightHand robotics). The hand combines an underlying capability for underactuated grasping [81] with sufficient degrees-of-actuation for the manipulation tasks set by the ARM-H program [80] (5 for the iHY Hand, reduced to 4 for the Model O).

Integration of the TacTip with the OpenHands (Table III) began with the Model M2 [82], a relatively simple gripper with just one movable finger and a large immobile thumb that was replaced with the TacTip-M2 [69]: an elongated tactile sensor with a rectangular sensing surface (Figure 4, middle row). Next, the two-fingered GR2 gripper [83] was integrated [71], replacing each fingertip with the TacTip-GR2: a compact version of the TacTip body using a fisheye lens and small camera with a standard-sized tip. Recently, the OpenHand tactile integration has culminated with the Tactile Model $\mathrm{O}$ (T-MO) [50]: the three fingertips were each replaced with a miniaturized TacTip, offering a low-cost 3D-printed dexterous robot hand with multipurpose soft optical tactile sensing.

A key question when considering the integration of tactile sensing into robotic systems is: what new capabilities are given by the sense of touch? For the 2-fingered tactile OpenHands (T-M2 and T-GR2), the integrated tactile sense enabled precise in-hand manipulation for unknown held objects [69], [71]. The 
fingers could roll an object to a desired location or along a trajectory over the tactile fingertip to millimetre-accuracy [6]. The 3-fingered T-MO's capabilities are based around the hand's high capability at grasping. Applying supervised deep learning to the tactile images of grasped objects gave accurate object classification (93\%, 26 objects) and grasp success prediction for lifting (95\%, same objects) [50]. Furthermore, the application of slip-detection methods enabled the hand to quickly re-grasp slipping objects (11 objects; 6 novel, 1 compliant) before being dropped [36] (see Section VI-B). The tactile hand could also prevent an object being dropped when weight was added, e.g. rice poured into a held tube, and lift an object on the first attempt with minimal grasp force [36].

Two other state-of-the-art hands have also been integrated with TacTips (Table III): the Shadow Smart Modular Grasper and the Pisa/IIT SoftHand. The Smart Modular Grasper is a fully-actuated dexterous 3-fingered hand that was combined with a compact TacTip using a wide-angle camera lens and customized body and tip from the 2016/2018 TacTip, which has been applied to stabilizing grasps by controlling the fingertip contacts [74], [84]. The SoftHand is an underactuated anthropomorphic robot hand designed around the principle of adaptive synergies from human hand movements [85]. This 5fingered hand has been integrated with a miniaturized TacTipSoftH of similar size to a human fingertip [30], a key milestone in this family of tactile hands (Figure 4, middle-row). A slightly-larger TacTip with an event-based camera (NeuroTac) has also been integrated [42]. Both versions of the tactile SoftHand open new possibilities for the sensorimotor control of anthropomorphic robot hands with biomimetic touch.

\section{B. Progress in tactile capabilities}

The purpose of an artificial sense of touch is to impart new dexterous capabilities to robotic systems that physically interact with their surroundings. These capabilities are built on sensorimotor perception and control: perception to process the tactile sensations for inferring states of the environment relative to the sensor, such as the location or shape of an object feature; and control to change that state, such as to slide a fingertip delicately over a feature or manipulate an object in a desired manner. A range of tactile perception and control methods have been developed for the TacTip family of soft biomimetic optical tactile sensors and hands (Table IV).

Over the period 2015-19, most of the methods used time series of marker deflections from rest, as they gave a compressed representation of the tactile image that has a biomimetic analogue with mechanoreceptor activity [6], [34], [40], [41] (see Section IIII). The pins displacements are also easily visualised to help interpret the tactile sensing (Figure 22.

Over the same period, a perception method based on a histogram likelihood model over the marker displacements was used [40]. For control, the position of an object feature was predicted, such as a cylinder or edge relative to the sensor [34], applied to rolling manipulation [69], [71], [89] or tapping around 2D contours [38], [46], [72]. However, those methods did not extend well from discrete to continuous and dynamic environments. Consequently, techniques interpolating over continuously-labelled data were tried, such as Gaussian process regression to control sliding motion around $2 \mathrm{D}$ contours [91], [92] and polynomial regression to smoothly control a multi-fingered grasp [74]. The large amount of training data can be an issues in some circumstances, and so online learning using Gaussian Process latent variable models has also been explored [76], [95]. Techniques for dynamic environments were also developed, such as detecting and correcting object slippage [35], [36], [78] to high accuracy $>90 \%$ with a support-vector machine.

The progress in machine learning methods has steadily improved the TacTip accuracy. Initial performance of $\sim 1 \mathrm{~mm}$ for 1D localization [34], [40], [46] or rolling manipulation [69], [71], [89] has improved by an order-of-magnitude to 0.1$0.5 \mathrm{~mm}$ [86]. Therefore, while the first results demonstrated hyperacuity finer than the pin spacing [40], [48], the latest results demonstrate sensitivity at the level of pixels on the tactile image. Thus far, the best sensitivity with a TacTip has been to detect $\lesssim 10$ microns indentation from an ultrasound haptic display, using signal averaging over the pin deflections from multiple tactile measurements to reduce noise [94].

\section{PeRIOd III (2019-): DeEp LEARNING WITH THE TACTIP}

Recently, the capabilities of the TacTip have undergone a step-change with the adoption of deep learning over tactile images. This has enabled, for the first time, tactile interaction in real time with complex objects in 3D (Figure 5). Prior to deep learning, the capabilities were limited to basic demonstrations such as rolling objects in 1D or exploring around flat shapes in 2D (Table IV). The new dexterous capabilities are a step closer to those we possess as humans.

The main benefit of deep learning is to predict quantities of interest directly from the tactile images while being insensitive to unknown variations that might otherwise interfere with the predictions. Consequently, the most advanced robot dexterity with the TacTip (Table IV) has mainly used convolutional neural networks (ConvNets), following their earlier success with optical tactile sensors based on the GelSight [96]-[98]. In principle, the marker deflections (Section VI-B) could be used as a lower-dimensional input to a neural network, although in practise it has been simpler to avoid the additional image processing step by using the entire tactile image as input.

New tactile capabilities developed with the TacTip include: (a) pose-based servo control, where a tactile fingertip mounted on a robot arm slides delicately over unknown complex 3D objects (Figure 5a; [77], [86], [87]); (b) pushing manipulation of unknown objects using only tactile sensing and proprioceptive knowledge of where the sensor is positioned relative to a goal location (Figure 5b; [88]); (c) acquiring the novel skill of single-fingered typing on a braille keyboard, learning the identity of keys and how to navigate the keyboard from touch (Figure 55; [75]); (d) item recognition and grasp-success prediction upon using the tactile sense of the three fingertips of the Tactile Model-O (T-MO) hand (Figure 5 $5 \mathrm{~d}$; [50]); (e) inhand manipulation of unknown objects to a stable grasp 
(a) Pose-based Servo Control via Tactile Deep Learning [86], [87]

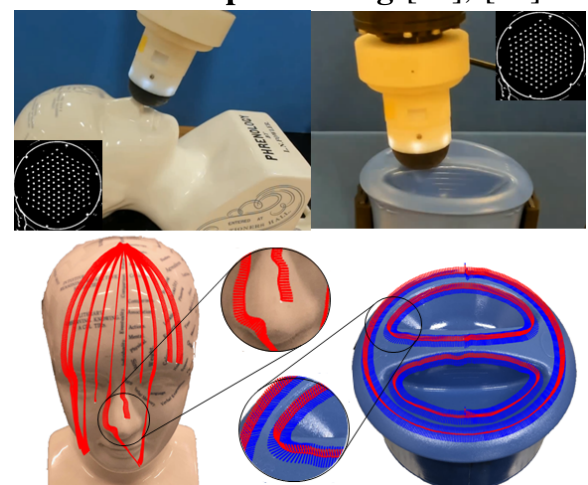

(d) Object \& grasp-success prediction with an underactuated tactile hand [50]

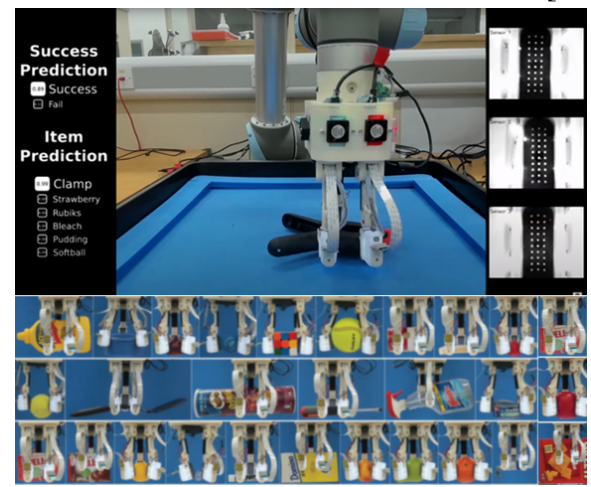

(b) Goal-based pushing via tactile \& proprioceptive feedback [88]

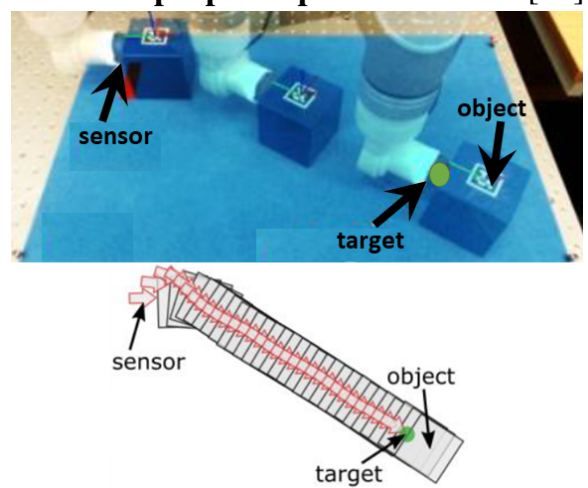

(e) In-hand tactile manipulation for fully-actuated stable grasping

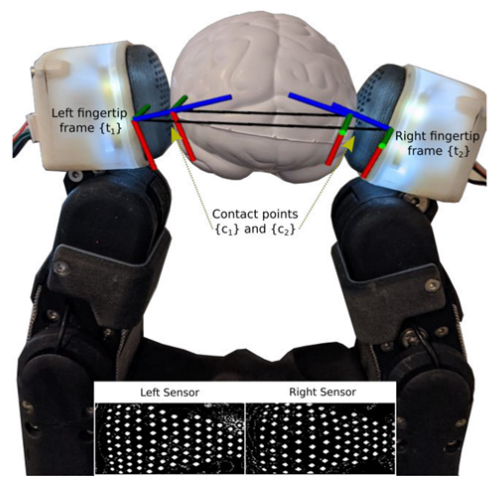

(c) Tactile Deep RL:

Learning to type Braille [75]

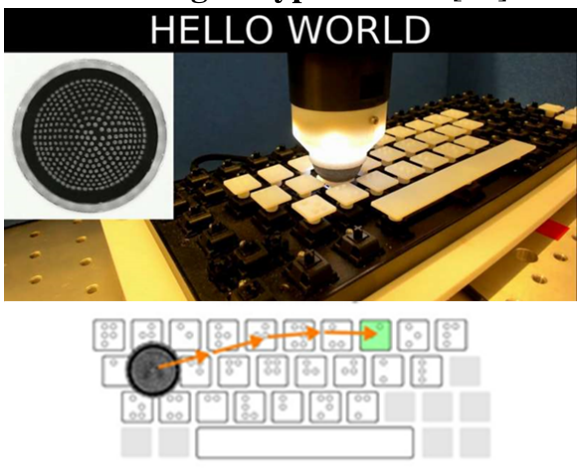

(f) Fine control of contact with an anthropomorphic tactile hand

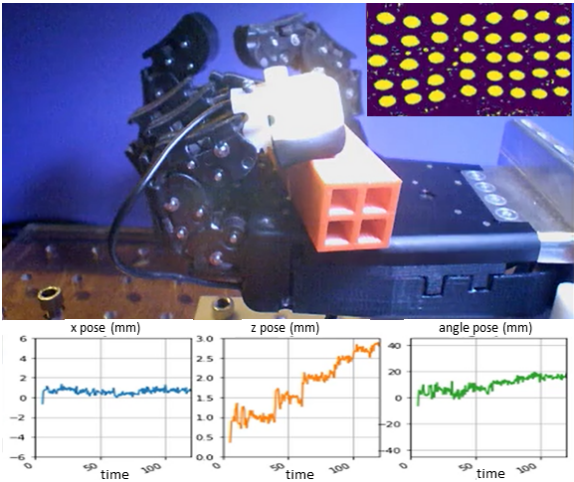

Fig. 5. Capabilities of deep learning with the BRL TacTip. The top row (a-c) shows examples of using a single tactile sensor mounted on a robot arm. The bottom row shows examples with tactile robot hands: (d) the Tactile Model O, (e) Tactile Modular Grasper and (f) the Pisa/IIT SoftHand. For all examples, the use of convolutional neural networks was critical for the tactile capability to be reached.

\begin{tabular}{|c|c|c|c|}
\hline Capability & Year & Accuracy & Robot \\
\hline 1D localization & 2015 & $0.7 \mathrm{~mm}$ & TacTip on ABB arm \\
\hline Rolling manipulation & 2016 & $\sim 1 \mathrm{~mm}$ & TacTip on ABB arm \\
\hline Gap measurement & 2016 & $0.5 \mathrm{~mm}$ & TacTip on ABB arm \\
\hline Rolling manipulation & 2016 & $\sim 1 \mathrm{~mm}$ & Tactile Model M2 hand \\
\hline 2D contour following (tapping) & 2017 & $\sim 1 \mathrm{~mm}, \sim 10 \mathrm{deg}$ & TacTip on ABB arm \\
\hline Rolling manipulation & 2017 & $\sim 1 \mathrm{~mm}$ & Tactile Model GR2 hand \\
\hline 1D localization; curvature estimation & 2017 & $0.1 \mathrm{~mm} ; \sim 1 \mathrm{~mm}$ & TacTip-FP1 on ABB arm \\
\hline Slip detection & 2018 & $>95 \%$ & TacTip on UR5 arm \\
\hline 2D contour following (sliding) & 2019 & $\sim 1 \mathrm{~mm}, \sim 10 \mathrm{deg}$ & TacTip on ABB arm \\
\hline 2D contour following (sliding) & 2019 & $\sim 1 \mathrm{~mm}, \sim 10 \mathrm{deg}$ & TacTip on ABB arm \\
\hline Grasp control (centre of contact) & 2019 & $\sim 1 \mathrm{deg}$ & Tactile Modular Grasper \\
\hline 1D localization; Normal force & 2019 & $\sim 0.5 \mathrm{~mm}, \sim 0.2 \mathrm{~N}$ & TacTip on ABB arm \\
\hline $\begin{array}{l}\text { Object recognition } \\
\text { Grasp success prediction }\end{array}$ & 2020 & $\begin{array}{l}>95 \% \\
>90 \%\end{array}$ & Tactile Model O hand \\
\hline Slip detection & 2020 & $>90 \%$ & Tactile Model O hand \\
\hline Ultrasound detection & 2020 & $\lesssim 10$ micron & TacTip on ABB arm \\
\hline $\begin{array}{l}\text { 3D surface localization } \\
\text { (depth, roll/pitch) }\end{array}$ & 2020 & $\begin{array}{l}0.1 \mathrm{~mm} \\
0.3 \mathrm{deg}\end{array}$ & TacTip on ABB arm \\
\hline $\begin{array}{l}\text { 3D edge localization } \\
\text { (horizontal, depth, roll/pitch, yaw) }\end{array}$ & 2020 & $\begin{array}{l}0.3 \mathrm{~mm}, 0.2 \mathrm{~mm} \\
1-2 \mathrm{deg}, 4 \mathrm{deg}\end{array}$ & TacTip on ABB arm \\
\hline Grasp control (stable pinch) & 2021 & $0.1 \mathrm{~mm}, 0.4 \mathrm{deg}$ & Tactile Modular Grasper \\
\hline $\begin{array}{l}\text { 3D surface following (sliding) } \\
\text { 3D contour following (sliding) }\end{array}$ & 2021 & $\begin{array}{l}0.1-0.5 \mathrm{~mm} \\
1-5 \mathrm{deg}\end{array}$ & TacTip on ABB arm \\
\hline 2D pushing (tapping motion) & 2021 & $\sim 1 \mathrm{~mm}$ & TacTip on UR5 arm \\
\hline
\end{tabular}

TABLE IV probabilistic classifier on pin displacements [34], [40] probabilistic classifier on pin displacements [89] probabilistic classifier on pin displacements [90] probabilistic classifier on pin displacements [69] probabilistic classifier on pin displacements [46] probabilistic classifier on pin displacements [71] probabilistic classifier on pin displacements [41]

SVM classifier on pin velocities [35]

PCA \& GP regressor on pin displacements [91], [92]

ConvNet regressor on $128 \times 128$ tactile image [77] polynomial regressor on pin displacements [74]

ConvNet autoencoder on ] $32 \times 32$ tactile image [93]

ConvNet classifier on $60 \times 120$ tactile image [50]

SVM or $\log$ Reg classifier on pin velocities [35]

GP regressor on pin displacements [94]

ConvNet regressor on $128 \times 128$ tactile image [86], [87]

ConvNet regressor on $128 \times 128$ tactile image [86], [87] servo control \& ConvNet on $128 \times 128$ tactile image [84] pose-based tactile servo control \&

ConvNet on $128 \times 128$ tactile image [86], [87] servo control \& ConvNet on $128 \times 128$ tactile image [88]

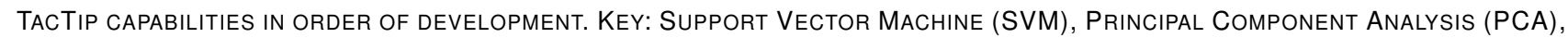
Gaussian Process (GP), Convolutional Neural Network (ConvNet), Logistic Regression (LogReg). The ABB arm is an IRB120

6-DoF INDUSTRIAL ROBOT; THE UR5 ARM IS A UNIVERSAL ROBOTICS 6-DoF ARM. TACTILE HANDS ARE DESCRIBED IN TABLE III] 
configuration with a fully-actuated tactile Shadow Modular Grasper (Figure 5e; [84]); (f) fine control of contact onto unknown objects placed in-hand using an anthropomorphic tactile hand based on the Pisa/IIT SoftHand (Figure 5; [30]). Humans can do all of these tasks with our sense of touch, which deep learning has enabled tactile robots to perform.

Why are ConvNets so useful when applied to tactile images from a soft biomimetic optical tactile sensor? Considering all the tasks in Figure 5, several reasons emerge:

(i) Ease of deployment - in all tasks, the trained neural network was applied directly with only basic pre-processing steps such as cropping, thresholding or concatenating tactile images. This simplifies the algorithmic pipeline and leaves less room for software or other issues to emerge.

(ii) Robustness - once trained, the predictions were relatively unaffected by uncontrolled variations that could cause issues, such as changes in ambient and internal lighting, wear of the skin, visible dust inside the sensor, and even after damage such as a (glued) tear in the TacTip skin after accidental crushing. (iii) Ease of scalability - the methods seem to extend straightforwardly from simple test scenarios to more complex realistic situations; for example, 2D servo control using a ConvNet with two pose variables extended relatively easily to $3 \mathrm{D}$ control with more pose variables [77], [86].

(iv) Generalization - the predictions seemed to be accurate even after large variations in the stimulus or task conditions; for example, ConvNets trained on simple stimuli, such as the pose of a planar surface or straight edge, maintained predictive performance on curved complex stimuli, enabling novel objects to be explored or manipulated [87], [88].

Going forward, a key question for deep learning with optical tactile sensing will be on the appropriate learning algorithm to acquire tactile skills. All but one of the tasks in Figure 5 trained the neural network model with supervised learning. However, a complementary approach is to use tactile deep $\mathrm{RL}$ with a reward signal that indicates the desired interactions with a physical environment. The third task (Figure 5d) successfully applied this approach to learning to type with a braille keyboard [75], leading to a policy network that could efficiently navigate the keyboard to press a desired key. Our expectation is that all considered tasks could also be learnt using deep RL, but for sample efficiency much of this learning should take place in a simulated tactile environment [99].

\section{CONCLUSION}

There are fundamental problems to be addressed in intelligent robotic interaction with complex environments that once solved will open up many application areas across engineering and robotics. One key problem is that there is a huge gap between what is achievable in research laboratories investigating robot manipulation and what is known about human dexterity and our sense of touch. Research on the TacTip aims to bridge that gap as an example of a SoftBOT sensor, combining Soft, Biomimetic, Optical and Tactile sensing.

SoftBOT sensors offer the opportunity to artificially recreate key aspects of the human sense of touch and our manual intelligence. This aim has two interconnected goals: (1) to advance knowledge of how our sense of touch leads to haptic intelligence from embodying those capabilities in robots; and (2) to improve the intelligent dexterity of robots with accessible robot hardware and software. Reaching human-like levels of dexterity has been the vision for industrial robotics since its roots in the 1950s, and is captured in the earlier origins of 'robot' from the Czech word robota for forced labour. Likewise, using biomimetic touch to achieve that goal has driven developments in robotic tactile sensing since the 1970s. A combination of advances in soft robotics, biomimetic tactile sensing and AI could enable that vision to become reality.

\section{ACKNOWLEDGMENTS}

I am fortunate to have the opportunity to work with many talented PhD students and postdocs at Bristol Robotics Laboratory, including Noor Alakhawand, Kirsty Aquilina, SophieAnne Baker, Thomas Cairnes, Alex Church, Pernilla Craig, Luke Cramphorn, Elena Giannaccini, Kipp McAdam Freud, Michele Garibo, Thom Griffith, Anupam Gupta, Fliss Inkpen, Jasper James, Haoran Li, Yijiong Lin, John Lloyd, Fraser MacDonald, Dabal Pedamonti, Nick Pestell, Efi Psomopoulou, Lizzie Stone, Emma Roscow and Ben Ward-Cherrier.

\section{References}

[1] J. Kevin O'Regan. Why Red Doesn't Sound Like a Bell: Understanding the Feel of Consciousness. Oxford University Press, 2011.

[2] Yuval Noah Harari. Homo Deus: A Brief History of Tomorrow. Random House, September 2016.

[3] Micah K. Johnson and Edward H. Adelson. Retrographic sensing for the measurement of surface texture and shape. In 2009 IEEE Conference on Computer Vision and Pattern Recognition, pages 1070-1077, June 2009.

[4] Wenzhen Yuan, Siyuan Dong, and Adelson Adelson, Edward H. GelSight: High-Resolution Robot Tactile Sensors for Estimating Geometry and Force. Sensors, 17(12):2762, 2017.

[5] Craig Chorley, Chris Melhuish, Tony Pipe, and Jonathan Rossiter Development of a tactile sensor based on biologically inspired edge encoding. In 2009 International Conference on Advanced Robotics, pages 1-6, June 2009.

[6] Benjamin Ward-Cherrier, Nicholas Pestell, Luke Cramphorn, Benjamin Winstone, Maria Elena Giannaccini, Jonathan Rossiter, and Nathan F. Lepora. The TacTip Family: Soft Optical Tactile Sensors with 3DPrinted Biomimetic Morphologies. Soft Robotics, 5(2):216-227, April 2018.

[7] Zhanat Kappassov, Juan-Antonio Corrales, and Véronique Perdereau. Tactile sensing in dexterous robot hands - Review. Robotics and Autonomous Systems, 74:195-220, December 2015.

[8] Ravinder S. Dahiya, Giorgio Metta, Maurizio Valle, and Giulio Sandini. Tactile Sensing-From Humans to Humanoids. IEEE Transactions on Robotics, 26(1):1-20, February 2010.

[9] Mark R. Cutkosky, Robert D. Howe, and William R. Provancher. Force and Tactile Sensors. In Springer Handbook of Robotics, pages 455-476. Springer Berlin Heidelberg, Berlin, Heidelberg, 2008.

[10] Mark H. Lee and H. R. Nicholls. Review Article Tactile sensing for mechatronics-a state of the art survey. Mechatronics, 9(1):1-31, February 1999.

[11] Saleem Khan, Leandro Lorenzelli, and Ravinder S. Dahiya. Technologies for Printing Sensors and Electronics Over Large Flexible Substrates: A Review. IEEE Sensors Journal, 15(6):3164-3185, June 2015.

[12] Hongbo Wang, Massimo Totaro, and Lucia Beccai. Toward Perceptive Soft Robots: Progress and Challenges. Advanced Science, 5(9):1800541, September 2018.

[13] Ravinder S. Dahiya. E-Skin: From Humanoids to Humans [Point of View]. Proceedings of the IEEE, 107(2):247-252, February 2019.

[14] Nathan F Lepora, Paul Verschure, and Tony J Prescott. The state of the art in biomimetics. Bioinspiration \& Biomimetics, 8(1):013001, January 2013. 
[15] Rolf Pfeifer, Max Lungarella, and Fumiya Iida. The challenges ahead for bio-inspired 'soft' robotics. Communications of the ACM, 55(11):76-87, November 2012.

[16] Sangbae Kim, Cecilia Laschi, and Barry Trimmer. Soft robotics: A bioinspired evolution in robotics. Trends in Biotechnology, 31(5):287294, May 2013.

[17] Nicholas Wettels, Veronica J. Santos, Roland S. Johansson, and Gerald E. Loeb. Biomimetic Tactile Sensor Array. Advanced Robotics, 22(8):829-849, January 2008.

[18] Clementine M. Boutry, Marc Negre, Mikael Jorda, Orestis Vardoulis, Alex Chortos, Oussama Khatib, and Zhenan Bao. A hierarchically patterned, bioinspired e-skin able to detect the direction of applied pressure for robotics. Science Robotics, 3(24):eaau6914, November 2018.

[19] Alexander C. Abad and Anuradha Ranasinghe. Visuotactile Sensors With Emphasis on GelSight Sensor: A Review. IEEE Sensors Journal, 20(14):7628-7638, July 2020.

[20] Thomas G. Strickler. Design of an optical touch sensing system for a remote manipulator, 1966.

[21] Kazuhiro Shimonomura. Tactile Image Sensors Employing Camera: A Review. Sensors, 19(18):3933, September 2019.

[22] Kazuto Kamiyama, Kevin Vlack, Terukazu Mizota, Hiroyuki Kajimoto, Naoki Kawakami, and Susumu Tachi. Vision-based sensor for realtime measuring of surface traction fields. IEEE Computer Graphics and Applications, 25(1):68-75, January 2005

[23] Wenzhen Yuan, Rui Li, Mandayam A. Srinivasan, and Edward H. Adelson. Measurement of shear and slip with a GelSight tactile sensor. In 2015 IEEE International Conference on Robotics and Automation (ICRA), pages 304-311, May 2015.

[24] Xi Lin and Michael Wiertlewski. Sensing the Frictional State of a Robotic Skin via Subtractive Color Mixing. IEEE Robotics and Automation Letters, 4(3):2386-2392, July 2019.

[25] Alex Krizhevsky, Ilya Sutskever, and Geoffrey E. Hinton. ImageNet classification with deep convolutional neural networks. Communications of the ACM, 60(6):84-90, May 2017.

[26] Daniel F. Gomes, Zhonglin Lin, and Shan Luo. GelTip: A Fingershaped Optical Tactile Sensor for Robotic Manipulation. 2020 IEEE/RSJ International Conference on Intelligent Robots and Systems (IROS), October 2020

[27] Mike Lambeta, Po-Wei Chou, Stephen Tian, Brian Yang, Benjamin Maloon, Victoria Rose Most, Dave Stroud, Raymond Santos, Ahmad Byagowi, Gregg Kammerer, Dinesh Jayaraman, and Roberto Calandra. DIGIT: A Novel Design for a Low-Cost Compact High-Resolution Tactile Sensor With Application to In-Hand Manipulation. IEEE Robotics and Automation Letters, 5(3):3838-3845, July 2020.

[28] Akhil Padmanabha, Frederik Ebert, Stephen Tian, Roberto Calandra, Chelsea Finn, and Sergey Levine. OmniTact: A Multi-Directional High Resolution Touch Sensor. arXiv:2003.06965 [cs], March 2020.

[29] Branden Romero, Filipe Veiga, and Edward H. Adelson. Soft, Round, High Resolution Tactile Fingertip Sensors for Dexterous Robotic Manipulation. arXiv:2005.09068 [cs], 2020.

[30] Nathan F. Lepora, Andrew Stinchcombe, Chris Ford, Alfred Brown, John Lloyd, Manuel G Catalano, Matteo Bianchi, and Benjamin WardCherrier. Towards integrated tactile sensorimotor control in anthropomorphic soft robotic hands. In IEEE International Conference on Robotics and Automation (ICRA), 2021.

[31] Roberta L. Klatzky and Susan J. Lederman. Touch. In Handbook of Psychology, pages 147-176. American Cancer Society, 2003.

[32] Victoria E. Abraira and David D. Ginty. The Sensory Neurons of Touch. Neuron, 79(4):618-639, August 2013.

[33] Amanda Zimmerman, Ling Bai, and David D. Ginty. The gentle touch receptors of mammalian skin. Science, 346(6212):950-954, November 2014.

[34] Nathan F. Lepora. Biomimetic Active Touch with Fingertips and Whiskers. IEEE Transactions on Haptics, 9(2):170-183, April 2016.

[35] Jasper Wollaston James, Nicholas Pestell, and Nathan F. Lepora. Slip Detection With a Biomimetic Tactile Sensor. IEEE Robotics and Automation Letters, 3(4):3340-3346, October 2018.

[36] Jasper Wollaston James and Nathan F. Lepora. Slip Detection for Grasp Stabilization With a Multifingered Tactile Robot Hand. IEEE Transactions on Robotics, pages 1-14, 2020.

[37] Benjamin Winstone, Gareth Griffiths, Tony Pipe, Chris Melhuish, and Jonathon Rossiter. TACTIP - Tactile Fingertip Device, Texture Analysis through Optical Tracking of Skin Features. In Biomimetic and Biohybrid Systems, volume 8064, pages 323-334. Springer Berlin Heidelberg, 2013.
[38] Nicholas Pestell, John Lloyd, Jonathan Rossiter, and Nathan F. Lepora. Dual-Modal Tactile Perception and Exploration. IEEE Robotics and Automation Letters, 3(2):1033-1040, April 2018.

[39] Gabor Soter, Andrew Conn, Helmut Hauser, Nathan F. Lepora, and Jonathan Rossiter. MultiTip: A multimodal mechano-thermal soft fingertip. In 2018 IEEE International Conference on Soft Robotics (RoboSoft), pages 239-244, Liverno, April 2018.

[40] Nathan F. Lepora and Benjamin Ward-Cherrier. Superresolution with an optical tactile sensor. In 2015 IEEE/RSJ International Conference on Intelligent Robots and Systems (IROS), pages 2686-2691, Hamburg, Germany, September 2015. IEEE.

[41] Luke Cramphorn, Benjamin Ward-Cherrier, and Nathan F. Lepora. Addition of a Biomimetic Fingerprint on an Artificial Fingertip Enhances Tactile Spatial Acuity. IEEE Robotics and Automation Letters, 2(3):1336-1343, July 2017.

[42] Benjamin Ward-Cherrier, Nicholas Pestell, and Nathan F. Lepora. NeuroTac: A Neuromorphic Optical Tactile Sensor applied to Texture Recognition. In 2020 IEEE International Conference on Robotics and Automation (ICRA), pages 2654-2660, Paris, France, May 2020.

[43] Benjamin Ward-Cherrier, Jorg Conradt, Manuel Giuseppe Catalano, Matteo Bianchi, and Nathan Lepora. A Miniaturised Neuromorphic Tactile Sensor Integrated with an Anthropomorphic Robot Hand. In 2020 IEEE/RSJ International Conference on Intelligent Robots and Systems (IROS), page 7, October 2020

[44] N. Cauna. Nature and functions of the papillary ridges of the digital skin. The Anatomical Record, 119(4):449-468, August 1954.

[45] Hannes P. Saal and Sliman J. Bensmaia. Touch is a team effort: Interplay of submodalities in cutaneous sensibility. Trends in Neurosciences, 37(12):689-697, December 2014.

[46] Nathan F. Lepora, Kirsty Aquilina, and Luke Cramphorn. Exploratory Tactile Servoing With Active Touch. IEEE Robotics and Automation Letters, 2(2):1156-1163, April 2017.

[47] Jack. M. Loomis. An Investigation of Tactile Hyperacuity. Sensory Processes, 3:289-302, 1980.

[48] Nathan F. Lepora, Uriel Martinez-Hernandez, Mathew Evans, Lorenzo Natale, Giorgio Metta, and Tony J. Prescott. Tactile Superresolution and Biomimetic Hyperacuity. IEEE Transactions on Robotics, 31(3):605618, June 2015.

[49] Seoung-Mok Yum, In-Keun Baek, Dongpyo Hong, Juhan Kim, Kyunghoon Jung, Seontae Kim, Kihoon Eom, Jeongmin Jang, Seonmyeong Kim, Matlabjon Sattorov, Min-Geol Lee, Sungwan Kim, Michael J. Adams, and Gun-Sik Park. Fingerprint ridges allow primates to regulate grip. Proceedings of the National Academy of Sciences, 117(50):31665-31673, December 2020.

[50] Jasper W. James, Alex Church, Luke Cramphorn, and Nathan F. Lepora. Tactile Model O: Fabrication and Testing of a 3D-Printed, ThreeFingered Tactile Robot Hand. Soft Robotics, page soro.2020.0019, December 2020.

[51] Alex Chortos, Jia Liu, and Zhenan Bao. Pursuing prosthetic electronic skin. Nature Materials, 15(9):937-950, September 2016.

[52] Zhengkun Yi, Yilei Zhang, and Jan Peters. Biomimetic tactile sensors and signal processing with spike trains: A review. Sensors and Actuators A: Physical, 269:41-52, January 2018.

[53] Carver Mead. Neuromorphic electronic systems. Proceedings of the IEEE, 78(10):1629-1636, October 1990.

[54] Alexander Schmitz, Perla Maiolino, Marco Maggiali, Lorenzo Natale, Giorgio Cannata, and Giorgio Metta. Methods and Technologies for the Implementation of Large-Scale Robot Tactile Sensors. IEEE Transactions on Robotics, 27(3):389-400, June 2011.

[55] Nicola J. Ferrier and Roger W. Brockett. Reconstructing the Shape of a Deformable Membrane from Image Data. The International Journal of Robotics Research, 19(9):795-816, September 2000.

[56] Kazuto Kamiyama, Hiroyuki Kajimoto, Naoki Kawakami, and Susumu Tachi. Evaluation of a vision-based tactile sensor. In IEEE International Conference on Robotics and Automation (ICRA), pages 1542-1547 Vol.2, New Orleans, LA, USA, 2004.

[57] Jonathan Platkiewicz, Hod Lipson, and Vincent Hayward. Haptic Edge Detection Through Shear. Scientific Reports, 6(1):23551, September 2016.

[58] Craig Chorley, Chris Melhuish, Tony Pipe, and Jonathan Rossiter. Tactile edge detection. In 2010 IEEE Sensors, pages 2593-2598, Kona, HI, November 2010.

[59] Benjamin Winstone, Gareth Griffiths, Chris Melhuish, Tony Pipe, and Jonathan Rossiter. TACTIP - Tactile fingertip device, challenges in reduction of size to ready for robot hand integration. In 2012 IEEE International Conference on Robotics and Biomimetics (ROBIO), pages 160-166, December 2012. 
[60] Ryo Kikuuwe, Akihito Sano, Hiromi Mochiyama, Naoyuki Takesue, K. Tsunekawa, S. Suzuki, and Hideo Fujimoto. The tactile contact lens. In Proceedings of IEEE Sensors, pages 535-538, Vienna, Austria, 2004.

[61] Ryo Kikuuwe, Akihito Sano, Hiromi Mochiyama, Naoyuki Takesue, and Hideo Fujimoto. Enhancing haptic detection of surface undulation. ACM Transactions on Applied Perception, 2(1):46-67, January 2005.

[62] Mitsuhito Ando, Hiromi Mochiyama, Toshinobu Takei, and Hideo Fujimoto. Effect of tactile contact lens on rubber artificial skin layer with a strain gauge. In 2016 IEEE/SICE International Symposium on System Integration (SII), pages 397-402, Sapporo, Japan, December 2016.

[63] Tareq Assaf, Craig Chorley, Jonathan Rossiter, Tony Pipe, Cesare Stefanini, and Chris Melhuish. Realtime processing of a biologically inspired tactile sensor for edge following and shape recognition. In TAROS, pages 24-30, 2010.

[64] Tareq Assaf, Calum Roke, Jonathan Rossiter, Tony Pipe, and Chris Melhuish. Seeing by Touch: Evaluation of a Soft Biologically-Inspired Artificial Fingertip in Real-Time Active Touch. Sensors, 14(2):25612577, February 2014.

[65] Calum Roke, Chris Melhuish, Tony Pipe, David Drury, and Craig Chorley. Deformation-Based Tactile Feedback Using a BiologicallyInspired Sensor and a Modified Display. In Towards Autonomous Robotic Systems, Lecture Notes in Computer Science, pages 114-124, Berlin, Heidelberg, 2011. Springer.

[66] Calum Roke, Chris Melhuish, Tony Pipe, David Drury, and Craig Chorley. Lump localisation through a deformation-based tactile feedback system using a biologically inspired finger sensor. Robotics and Autonomous Systems, 60(11):1442-1448, November 2012.

[67] Calum Roke, Adam Spiers, Tony Pipe, and Chris Melhuish. The effects of laterotactile information on lump localization through a teletaction system. In 2013 World Haptics Conference (WHC), pages 365-370, Daejeon, April 2013.

[68] Benjamin Ward-Cherrier, Nicholas Pestell, Luke Cramphorn, and Nathan F. Lepora. TacTip. In Soft Robotics Toolkit. https://softroboticstoolkit.com/tactip, 2016.

[69] Benjamin Ward-Cherrier, Luke Cramphorn, and Nathan F. Lepora. Tactile Manipulation With a TacThumb Integrated on the Open-Hand M2 Gripper. IEEE Robotics and Automation Letters, 1(1):169-175, January 2016.

[70] Benjamin Winstone, Chris Melhuish, Tony Pipe, Mark Callaway, and Sanja Dogramadzi. Toward Bio-Inspired Tactile Sensing Capsule Endoscopy for Detection of Submucosal Tumors. IEEE Sensors Journal, 17(3):848-857, February 2017.

[71] Benjamin Ward-Cherrier, Nicolas Rojas, and Nathan F. Lepora. ModelFree Precise in-Hand Manipulation with a 3D-Printed Tactile Gripper. IEEE Robotics and Automation Letters, 2(4):2056-2063, October 2017.

[72] Benjamin Ward-Cherrier, Luke Cramphorn, and Nathan F. Lepora. Exploiting Sensor Symmetry for Generalized Tactile Perception in Biomimetic Touch. IEEE Robotics and Automation Letters, 2(2):12181225, April 2017.

[73] Nathan F. Lepora, Martin Pearson, and Luke Cramphorn. TacWhiskers: Biomimetic Optical Tactile Whiskered Robots. In 2018 IEEE/RSJ International Conference on Intelligent Robots and Systems (IROS), pages 7628-7634, Madrid, October 2018.

[74] Nicholas Pestell, Luke Cramphorn, Fotios Papadopoulos, and Nathan F. Lepora. A Sense of Touch for the Shadow Modular Grasper. IEEE Robotics and Automation Letters, 4(2):2220-2226, April 2019.

[75] Alex Church, John Lloyd, Raia Hadsell, and Nathan F. Lepora. Deep Reinforcement Learning for Tactile Robotics: Learning to Type on a Braille Keyboard. IEEE Robotics and Automation Letters, 5(4):61456152, October 2020.

[76] Elizabeth Anne Stone, Nathan Lepora, and David A W Barton. Walking on TacTip Toes: A Tactile Sensing Foot for Walking Robots. 2020 IEEE/RSJ International Conference on Intelligent Robots and Systems (IROS), page 7, 2020.

[77] Nathan F. Lepora, Alex Church, Conrad de Kerckhove, Raia Hadsell, and John Lloyd. From Pixels to Percepts: Highly Robust Edge Perception and Contour Following Using Deep Learning and an Optical Biomimetic Tactile Sensor. IEEE Robotics and Automation Letters, 4(2):2101-2107, April 2019.

[78] Jasper Wollaston James, Stephen Redmond, and Nathan Lepora. A Biomimetic Tactile Fingerprint Induces Incipient Slip. In 2020 IEEE/RSJ International Conference on Intelligent Robots and Systems (IROS), pages 9833-9839, October 2020.

[79] Raymond Ma and Aaron Dollar. Yale OpenHand Project: Optimizing Open-Source Hand Designs for Ease of Fabrication and Adoption. IEEE Robotics \& Automation Magazine, 24(1):32-40, March 2017.
[80] Lael U. Odhner, Leif P. Jentoft, Mark R. Claffee, Nicholas Corson, Yaroslav Tenzer, Raymond R. Ma, Martin Buehler, Robert Kohout, Robert D. Howe, and Aaron M. Dollar. A compliant, underactuated hand for robust manipulation. The International Journal of Robotics Research, 33(5):736-752, April 2014.

[81] Aaron M. Dollar and Robert D. Howe. The Highly Adaptive SDM Hand: Design and Performance Evaluation. The International Journal of Robotics Research, 29(5):585-597, April 2010.

[82] Raymond R. Ma, Adam Spiers, and Aaron M. Dollar. M2 Gripper: Extending the Dexterity of a Simple, Underactuated Gripper. In Advances in Reconfigurable Mechanisms and Robots II, volume 36, pages 795-805. Springer International Publishing, Cham, 2016.

[83] Nicolas Rojas, Raymond R. Ma, and Aaron M. Dollar. The GR2 Gripper: An Underactuated Hand for Open-Loop In-Hand Planar Manipulation. IEEE Transactions on Robotics, 32(3):763-770, June 2016.

[84] Efi Psomopoulou, Nicholas Pestell, Fotios Papadopoulos, John Lloyd, Zoe Doulgeri, and Nathan F Lepora. A robust controller for stable 3D pinching using tactile sensing. page 8 .

[85] M. G. Catalano, G. Grioli, A. Farnioli, A. Serio, C. Piazza, and A. Bicchi. Adaptive synergies for the design and control of the Pisa/IIT SoftHand. The International Journal of Robotics Research, 33(5):768782,2014

[86] Nathan F. Lepora and John Lloyd. Optimal Deep Learning for Robot Touch: Training Accurate Pose Models of 3D Surfaces and Edges. IEEE Robotics \& Automation Magazine, 27(2):66-77, June 2020.

[87] Nathan F. Lepora and John Lloyd. Pose-Based Servo Control with Soft Tactile Sensing. December 2020.

[88] John Lloyd and Nathan F Lepora. Goal-Driven Robotic Pushing Using Tactile and Proprioceptive Feedback. IEEE Transactions on Robotics, 2020.

[89] Luke Cramphorn, Benjamin Ward-Cherrier, and Nathan F. Lepora. Tactile manipulation with biomimetic active touch. In 2016 IEEE International Conference on Robotics and Automation (ICRA), pages 123-129, Stockholm, Sweden, May 2016. IEEE.

[90] Nathan F. Lepora and Benjamin Ward-Cherrier. Tactile Quality Control With Biomimetic Active Touch. IEEE Robotics and Automation Letters, 1(2):646-652, July 2016.

[91] Kirsty Aquilina, David A. W. Barton, and Nathan F. Lepora. Principal Components of Touch. In 2018 IEEE International Conference on Robotics and Automation (ICRA), pages 4071-4078, Brisbane, QLD, May 2018. IEEE.

[92] Kirsty Aquilina, David A. W. Barton, and Nathan F. Lepora. Shearinvariant Sliding Contact Perception with a Soft Tactile Sensor. In 2019 International Conference on Robotics and Automation (ICRA), pages 4283-4289, Montreal, Canada, May 2019.

[93] Marsela Polic, Ivona Krajacic, Nathan Lepora, and Matko Orsag. Convolutional Autoencoder for Feature Extraction in Tactile Sensing. IEEE Robotics and Automation Letters, 4(4):3671-3678, October 2019.

[94] Noor Alakhawand, William Frier, Kipp McAdam Freud, Orestis Georgiou, and Nathan F. Lepora. Sensing Ultrasonic Mid-Air Haptics with a Biomimetic Tactile Fingertip. In Haptics: Science, Technology, Applications, volume 12272, pages 362-370. Springer International Publishing, Cham, 2020.

[95] Elizabeth A Stone, Nathan F Lepora, and David A W Barton. Learning to Live Life on the Edge: Online Learning for Data-Efficient Tactile Contour Following. In 2020 IEEE/RSJ International Conference on Intelligent Robots and Systems (IROS), page 7, 2020.

[96] Wenzhen Yuan, Chenzhuo Zhu, Andrew Owens, Mandayam A. Srinivasan, and Edward H. Adelson. Shape-independent hardness estimation using deep learning and a GelSight tactile sensor. In 2017 IEEE International Conference on Robotics and Automation (ICRA), pages 951-958, Singapore, Singapore, May 2017.

[97] Roberto Calandra, Andrew Owens, Manu Upadhyaya, Wenzhen Yuan, Justin Lin, Edward H. Adelson, and Sergey Levine. The Feeling of Success: Does Touch Sensing Help Predict Grasp Outcomes? In Conference on Robot Learning, pages 314-323. PMLR, October 2017.

[98] Francois R. Hogan, Maria Bauza, Oleguer Canal, Elliott Donlon, and Alberto Rodriguez. Tactile Regrasp: Grasp Adjustments via Simulated Tactile Transformations. In 2018 IEEE/RSJ International Conference on Intelligent Robots and Systems (IROS), pages 2963-2970, Madrid, October 2018.

[99] Zihan Ding, Nathan F. Lepora, and Edward Johns. Sim-to-Real Transfer for Optical Tactile Sensing. In 2020 IEEE International Conference on Robotics and Automation (ICRA), pages 1639-1645, Paris, France, May 2020 . 